\title{
Distributed Multi-agent Optimization Subject to Nonidentical Constraints and Communication Delays *
}

\author{
Peng Lin ${ }^{\mathrm{a}, \mathrm{b}}$, Wei Ren ${ }^{\mathrm{c}}$, Yongduan Song d,e \\ ${ }^{a}$ School of Automation Engineering, University of Electronic Science and Technology of China, China \\ ${ }^{\mathrm{b}}$ State key Laboratory of Networking and Switching Technology, Beijing University of Posts and Telecommunications, Beijing, China \\ ${ }^{\mathrm{c}}$ Department of Electrical and Computer Engineering, University of California, Riverside, USA \\ ${ }^{\mathrm{d}}$ Key Laboratory of Dependable Service Computing, Cyber Physical Society of Ministry of Education, Chongqing University, China \\ ${ }^{\mathrm{e}}$ School of Automation, Chongqing University, China
}

\begin{abstract}
In this paper, we study a distributed optimization problem using a subgradient projection algorithm for multi-agent systems subject to nonidentical constraints and communication delays under local communication. Here the agents capable of communicating with their local neighbors are constrained to remain in possibly different closed convex sets and optimize a global objective function composed of a sum of local objective functions, each of which is known to only one agent. First, we consider the case of fixed graphs and show that distributed optimization might not be achieved on general strongly connected directed graphs. Instead, the agents optimize a weighted sum of the local objective functions. Then we consider the case of switching graphs and show that distributed optimization can be achieved when the adjacency matrices are doubly stochastic and the union of the directed graphs is strongly connected among each time interval of a certain bounded length. Furthermore, we consider the case of communication delays, where the delays are mutually independent. It is shown that the distributed optimization problem can be solved by introducing additional delays to the subgradient projection algorithm and the communication delays can be arbitrarily bounded. Finally, numerical examples are included to show the obtained theoretical results.
\end{abstract}

Key words: Distributed optimization, Multi-agent Systems, Cooperative Control, Switching topologies, Communication delays.

\section{Introduction}

Distributed optimization of multi-agent systems arises in a variety of distributed processing problems such as distributed estimation, distributed motion planning, distributed resource allocation, and distributed congestion control [1-14]. The objective is to solve a distributed optimization problem

\footnotetext{
* This paper was not presented at any IFAC meeting. This work was supported in part by the National Natrual Science Foundation of China (61203080, 61573082, 61528301, 61134001, 61120106010), the National Science Foundation (ECCS-1307678), the Foundation of State Key Laboratory of Networking and Switching Technology Foundation (SKLNST2011105, SKLNST2013109), and the Major State Basic Research Development Program 973 (No. 2012CB215202, No. 2014CB249200). Corresponding author W. Ren. Tel. +1 951-8276204. Fax +1 951-827-2425.

Email addresses: lin_peng@163.com (Peng Lin), ren@ee.ucr.edu (Wei Ren), ydsong@cqu.edu.cn (Yongduan Song).
}

where the global objective function is composed of a sum of local objective functions, each of which is known to only one agent. Recently, many results on distributed optimization problems have been obtained under various assumptions on network topologies and agent states. Distributed optimization problems are first studied systematically in [1] when there are no state constraints. Here the union of the directed graphs are assumed to be strongly connected among each time interval of a certain bounded length and the adjacency matrices are doubly stochastic. A distributed subgradient method is introduced to solve the distributed optimization problem and error bounds on the objective functions are given. As a continuation of [1], a distributed subgradient projection algorithm is developed in [2] for distributed optimization where each agent is constrained to remain in a closed convex set. Here the convergence analysis is given in the case of identical closed convex sets and in the case of nonidentical closed convex sets but fully connected (all-to-all) graphs. Inspired by the works of [1] and [2], [3] and [4] consider a random environment when the agents have identical state constraints. In [5], nonidentical constraints are consid- 
ered but the analysis approach heavily relies on the property that the transition matrix is doubly stochastic. In [6], nonidentical constraints are also considered but it is assumed that the communication graph is undirected and each possible communication link is functioning with a given probability. Thus, the communication graph by taking the expectation is essentially fixed and undirected.

Different from [1-6], a dual averaging subgradient algorithm is developed and analyzed in [7] for randomized graphs under the assumption that all agents remain in the same closed convex set. It is shown that the number of iterations required by their algorithm scales inversely in the spectral gap of the network. Moreover, distributed optimization problems with asynchronous stepsizes or inequality-equality constraints or using other algorithms are studied in [8-14]. Corresponding conditions are given to ensure the system to converge to the optimal point or its neighborhood. However, in [7-14] it is assumed that the state sets of the agents are identical or the objective function finally converges to only a neighborhood of the optimal set. In addition, in practical applications, due to packet losses resulting in retransmission and asynchronous clocks of the agents, the states received from neighbors could be outdated, which would yield communication delays. When the communication delays exist, the balance and symmetry of the communication graphs would be destroyed, which makes the existing algorithms without delays (e.g. [1-14]) no longer valid. It is important to study the distributed optimization problem in the presence of communication delays.

In this paper, we study the distributed optimization problem using a subgradient projection algorithm for multi-agent systems subject to nonidentical constraints and communication delays under local communication in a more general setting to take into account more realistic challenges, expanding on our preliminary results presented in [15]. In contrast to the existing results (see $[2,4-6]$ ), where the constraint sets are assumed to be identical, the communication graphs are assumed to be fixed and fully connected, the approach heavily relies on the double stochasticity of the transition matrix, or the communication graph by taking the expectation is essentially fixed and undirected, we consider three more general cases, namely, a fixed general strongly connected graph, a switching directed graph, and the presence of communication delays. Our method is based on analyzing the relationship of the distances between each agent's state and its projection to the intersection of the constraint sets at any two consecutive time steps, estimating the values of these distances near their superior limit point by reverse analysis, and estimating the error between the sum of all agents's local objective functions and the optimal global objective function. Such an analysis is particularly useful for dealing with the asymmetry caused by both unbalanced graphs and communication delays and hence generalizes the existing results. The contributions of the current paper are three-fold. First, we consider the case of fixed graphs and show that distributed optimization might not be achieved on general strongly connected directed graphs. Instead, the agents opti- mize a weighted sum of the local objective functions. Then we consider the case of switching graphs and show that distributed optimization can be achieved when the adjacency matrices are doubly stochastic and the union of the directed graphs is strongly connected among each time interval of a certain bounded length. Furthermore, we consider the case of communication delays, where the delays are mutually independent. It is shown that the distributed subgradient optimization problem can be solved by introducing additional delays to the subgradient projection algorithm and the communication delays can be arbitrarily bounded.

Notations. The following notations will be used throughout this paper. $\mathbb{R}^{m}$ denotes the set of all $m$ dimensional real column vectors; $\mathcal{I}$ denotes the set $\{1,2, \cdots, n\} ; Z$ and $Z_{+}$ denote the sets of all nonnegative integers and all positive integers; $x^{T}$ and $A^{T}$ denote the transpose of the vector $x$ and the matrix $A ;|x|$ denotes the absolute value of the scalar $x ;\|x\|_{1}$ and $\|x\|$ denote the 1-norm and the standard Euclidean norm of the vector $x ; \mathbf{1}$ represents $[1, \cdots, 1]^{T}$ with compatible dimensions; $x^{i}$ denotes the $i$ th entry of a vector $x ;[A]_{j},[A]^{i}$ and $[A]_{j}^{i}$ denote the $j$ th column, the $i$ th row and the $(i, j)$ entry of a matrix $A$. Let $P_{Y}(\chi)$ be the projection of a vector $\chi$ on a closed convex set $Y$, i.e., $P_{Y}(\chi)=\arg \min _{\bar{\chi} \in Y}\|\chi-\bar{\chi}\|$.

\section{Preliminaries}

In this section, we first introduce some preliminary results about graph theory, nonnegative matrices, and convex analysis (see $[2,16,17])$.

Let $\mathcal{G}(\mathcal{I}, \mathcal{E}, \mathcal{A})$ be a graph of order $n$, where $\mathcal{I}$ is the set of nodes, $\mathcal{E} \subseteq \mathcal{I} \times \mathcal{I}$ is the set of edges, and $\mathcal{A}=\left[a_{j}^{i}\right] \in \mathbb{R}^{n \times n}$ is the weighted adjacency matrix ${ }^{1}$. An edge of $\mathcal{G}$ is denoted by $(i, j)$. Then the set of neighbors of node $i$ is denoted by $N_{i}=\{j \in \mathcal{I}:(j, i) \in \mathcal{E}\}$. We assume that $(i, i) \in \mathcal{E}$ and hence $i \in N_{i}$. The entry $a_{j}^{i}$ of the adjacency matrix $\mathcal{A}$, which denotes the edge weight associated with the edge $(j, i)$, is positive if $(j, i) \in \mathcal{E}$ and is zero otherwise. A directed path is a sequence of ordered edges of the form $\left(i_{1}, i_{2}\right),\left(i_{2}, i_{3}\right), \cdots$, where $i_{j} \in \mathcal{I}$ in a directed graph. A directed graph is strongly connected if there is a directed path from every node to every other node. A directed graph is balanced if $\sum_{j=1}^{n} a_{j}^{i}=$ $\sum_{j=1}^{n} a_{i}^{j}$ for all $i \in \mathcal{I}$. Moreover, a graph is undirected if $(i, j) \in \mathcal{E}$ implies $(j, i) \in \mathcal{E}$. An undirected graph is fully connected if there is an edge between every pair of distinct nodes. For undirected graphs, we assume $a_{j}^{i}=a_{i}^{j}$ for all $i, j \in \mathcal{I}$. Hence each undirected graph is balanced.

Given $C=\left[c_{j}^{i}\right] \in \mathbb{R}^{n \times r}, C$ is nonnegative $(C \geq 0)$ if all its entries $c_{j}^{i}$ are nonnegative, and $C$ is positive $(C>0)$ if all its entries $c_{j}^{i}$ are positive. Further, $C \geq D$ if $C-D \geq 0$,

\footnotetext{
$\overline{{ }^{1} \text { Here } a_{j}^{i}}$ denotes the $(i, j)$ entry of $\mathcal{A}$.
} 
and $C>D$ if $C-D>0$. A nonnegative matrix $C \in \mathbb{R}^{n \times n}$ is stochastic, if it is satisfies $C \mathbf{1}=\mathbf{1}$. A stochastic matrix $C \in \mathbb{R}^{n \times n}$ is doubly stochastic, if it satisfies $\mathbf{1}^{T} C=\mathbf{1}^{T}$.

Lemma 1 [2] Suppose that $Y \subseteq \mathbb{R}^{m}$ is a nonempty closed convex set. For any $\chi, \zeta \in \mathbb{R}^{m}$ and all $\xi \in Y, \| P_{Y}(\chi)-$ $\xi\left\|^{2} \leq\right\| \chi-\xi\left\|^{2}-\right\| P_{Y}(\chi)-\chi \|^{2}$ and $\left\|P_{Y}(\chi)-P_{Y}(\zeta)\right\| \leq$ $\|\chi-\zeta\|$.

Lemma 2 Suppose that $Y \subseteq \mathbb{R}^{m}$ is a nonempty closed convex set. Let $\chi^{i} \in \mathbb{R}^{m}, i \in \mathcal{I}$, be arbitrary vectors and let $a_{i} \geq 0, i \in \mathcal{I}$, be scalars such that $\sum_{i=1}^{n} a_{i}=1$. Then we have $\left\|\sum_{i=1}^{n} a_{i} \chi^{i}-P_{Y}\left(\sum_{i=1}^{n} a_{i} \chi^{i}\right)\right\| \leq \sum_{i=1}^{n} a_{i} \| \chi^{i}-$ $P_{Y}\left(\chi^{i}\right) \|$.

Proof: Since $P_{Y}\left(\chi^{i}\right) \in Y$ and $\sum_{i=1}^{n} a_{i} P_{Y}\left(\chi^{i}\right)$ is a convex combination of the vectors $P_{Y}\left(\chi^{i}\right)$, then $\sum_{i=1}^{n} a_{i} P_{Y}\left(\chi^{i}\right) \in Y$. Thus, from the definition of projection, it follows that $\left\|\sum_{i=1}^{n} a_{i} \chi^{i}-P_{Y}\left(\sum_{i=1}^{n} a_{i} \chi^{i}\right)\right\| \leq$ $\left\|\sum_{i=1}^{n} a_{i} \chi^{i}-\sum_{i=1}^{n} a_{i} P_{Y}\left(\chi^{i}\right)\right\| \leq \sum_{i=1}^{n} a_{i}\left\|\chi^{i}-P_{Y}\left(\chi^{i}\right)\right\|$.

\section{Problem Statement}

Consider a multi-agent system consisting of $n>1$ discretetime agents. Each agent is regarded as a node in the directed graph $\mathcal{G}(k)$. Each edge $(j, i) \in \mathcal{E}$ corresponds to an available information channel from agent $i$ to agent $j$. Each agent updates its current state based upon the information received from its neighbors, denoted by $N_{i}(k)$, at time $k$. The weight of edge $(i, j)$ denotes the reliability of the received information. The state of each agent $x^{i}(k) \in \mathbb{R}^{m}$ is constrained to lie in a nonempty closed convex set $X_{i} \subseteq \mathbb{R}^{m}$ which is known by only agent $i$. The agents' objective is to cooperatively solve the following optimization problem while remaining in their corresponding sets $X_{i}$ :

$$
\begin{array}{ll}
\operatorname{minimize} & \sum_{i=1}^{n} f_{i}(x) \\
\text { subject to } & x \in X \triangleq \bigcap_{i=1}^{n} X_{i},
\end{array}
$$

where each $f_{i}: \mathbb{R}^{m} \rightarrow \mathbb{R}$ represents the local objective function of agent $i$, which is convex and known by only agent $i$.

As stated in [2], $f_{i}$ is differentiable almost everywhere due to its convexity. At the points where the function is not differentiable, a subgradient can be used as a gradient. To be specific, for each convex function $f_{i}$ and a point $\bar{x}$, a subgradient of the function $f_{i}$ at $\bar{x}$ is a vector $s_{f_{i}}(\bar{x}) \in \mathbb{R}^{m}$ such that $f_{i}(\bar{x})+s_{f_{i}}^{T}(\bar{x})(x-\bar{x}) \leq f_{i}(x)$ for all $x$. For convenience of the subsequent discussion, we use $\partial f_{i}(\bar{x})$ to denote the set of all subgradients of $f_{i}(\bar{x})$.

To show the main results, the following assumptions are needed.
Assumption 1 [2] There exists a scalar $\delta>0$ and a vector $\bar{\chi} \in X$ such that $\{\xi \mid\|\xi-\bar{\chi}\| \leq \delta\} \subset X$.

Under Assumption 1, $X$ is a nonempty closed convex set and contains at least one interior point.

Lemma 3 [2] Let $\chi^{i} \in X_{i}, i \in \mathcal{I}$, be arbitrary vectors. Under Assumption 1, $\left\|\frac{1}{n} \sum_{i=1}^{n} \chi^{i}-P_{X}\left(\frac{1}{n} \sum_{i=1}^{n} \chi^{i}\right)\right\| \leq$ $\frac{1}{n \delta}\left(\sum_{i=1}^{n}\left\|\chi^{i}-\bar{\chi}\right\|\right)\left(\sum_{j=1}^{n}\left\|\frac{1}{n} \sum_{i=1}^{n} \chi^{i}-P_{X_{j}}\left(\frac{1}{n} \sum_{i=1}^{n} \chi^{i}\right)\right\|\right)$, where $\bar{\chi}$ and $\delta$ are defined in Assumption 1.

Assumption 2 There exists a positive constant $0<\eta<1$ such that for all $i, j \in \mathcal{I}$ and all $k \geq 0$ (a) $a_{i}^{i}(k) \geq \eta$; (b) $a_{j}^{i}(k) \geq \eta$ if $a_{j}^{i}(k)>0$.

Assumption 2 ensures that if an edge weight is nonzero, it is lower bounded by a positive number. This prevents the edge weight from going arbitrarily close to zero and guarantees that the information interaction works in a nonvanishing rate.

Assumption 3 For all $i, j \in \mathcal{I}$, (a) $\sum_{j=1}^{n} a_{j}^{i}(k)=1$; (b) $\sum_{j=1}^{n} a_{i}^{j}(k)=1$.

Assumption 3(a) is used to yield a convex combination of the agents' states in the distributed optimization algorithms shown later, while Assumption 3(b) is further used to balance the influence of each agent's state to the consensus value. If Assumptions 3(a) and 3(b) are both satisfied, the adjacency matrix $\mathcal{A}(k)$ at each time $k$ is doubly stochastic and the directed graph $\mathcal{G}(k)$ is hence balanced.

Assumption 4 There exists an infinite sequence of times $k_{0}, k_{1}, k_{2}, \cdots$, where $k_{0}=0,0<k_{j+1}-k_{j} \leq M, j \in$ $Z, M \in Z_{+}$, such that the union of the directed graphs $\mathcal{G}\left(k_{j}\right), \cdots, \mathcal{G}\left(k_{j+1}-1\right)$ is strongly connected.

Assumption 4 says that the directed graphs are jointly strongly connected in each interval $\left[k_{j}, k_{j+1}\right)$, which ensures that the agents can interact with each other directly or indirectly sufficiently often over time.

Assumption 5 Each $X_{i}$ is compact, i.e., there is a scalar $H>0$ such that $\|\chi\|<H$ for all $\chi \in X_{i}$ and all $i$.

Assumption 5 means that the agents' states cannot be arbitrarily large. Moreover, in view of the continuity and convexity of the local objective function $f_{i}(x)$, it can be obtained that, under Assumption 5, the subgradients of $f_{i}(x)$ are bounded on a bounded closed convex closure $\tilde{Y}$ such that $X_{i} \subset \tilde{Y}$ for all $i$, which is mathematically summarized in the following lemma.

Lemma 4 Let $a_{i} \geq 0, i \in \mathcal{I}$, be scalars such that $\sum_{i=1}^{n} a_{i}=1$. Then, under Assumption 5, there exists a scalar $L>0$ such that $\left\|s_{f_{i}}\left(\sum_{i=1}^{n} a_{i} x^{i}\right)\right\| \leq L$ for all $s_{f_{i}}\left(\sum_{i=1}^{n} a_{i} x^{i}\right) \in \partial f_{i}\left(\sum_{i=1}^{n} a_{i} x^{i}\right)$, all $i$ and all $x^{i} \in X_{i}$. 
Proof: Since each $X_{i}$ is compact, there must exist a compact convex set $\tilde{Y}_{1}$ such that $X_{i} \subset \tilde{Y}_{1}$ for all $i$. Note from [21] that the subgradients of a convex function are uniformly bounded over a bounded set. Thus, there exists a scalar $L>$ 0 such that $\left\|s_{f_{i}}\left(y_{1}\right)\right\| \leq L$ for all $s_{f_{i}}\left(y_{1}\right) \in \partial f_{i}\left(y_{1}\right)$, all $i$ and all $y_{1} \in \tilde{Y}_{1}$. Since $X_{i} \subset \tilde{Y}_{1}$ for all $i$, it follows that $\sum_{i=1}^{n} a_{i} x^{i} \in \tilde{Y}_{1}$ and hence $\left\|s_{f_{i}}\left(\sum_{i=1}^{n} a_{i} x^{i}\right)\right\| \leq L$ for all $s_{f_{i}}\left(\sum_{i=1}^{n} a_{i} x^{i}\right) \in \partial f_{i}\left(\sum_{i=1}^{n} a_{i} x^{i}\right)$, all $i$ and all $x^{i} \in X_{i}$.

Assumption 6 The step sizes, $\alpha_{k}$, satisfy that $\sum_{k=0}^{+\infty} \alpha_{k}=$ $+\infty$ and $\sum_{k=0}^{+\infty} \alpha_{k}^{2}<+\infty$.

This step-size assumption is used to guarantee the convergence of the distributed optimization algorithms shown later to the optimal point.

Remark 1 In Assumption 4, we do not assume that the union of the graphs has a directed spanning tree because there might be an agent that remains stationary in its corresponding set and all other agents cannot reach a consensus with the agent due to the state constraints.

\section{Distributed optimization problem with nonidentical constraints}

A subgradient projection algorithm is proposed in [2] as

$$
x^{i}(k+1)=P_{X_{i}}\left[\sum_{j \in N_{i}(k)} a_{j}^{i}(k) x^{j}(k)-\alpha_{k} d_{i}(k)\right],
$$

where $a_{j}^{i}(k)$ is the $(i, j)$ entry of the adjacency matrix $\mathcal{A}(k)$ at time $k, N_{i}(k)$ is the neighbor set of agent $i$ at time $k, \alpha_{k}>0$ is the step size at time $k$, and the vector $d_{i}(k)$ is a subgradient of the local objective function $f_{i}(x)$ at $\sum_{j \in N_{i}(k)} a_{j}^{i}(k) x^{j}(k)$. Here, it is assumed that $x_{i}(k)=$ $\omega_{i}(k)$ for all $k \leq 0$ for all $i$, where $\omega_{i}(k)$ is a discrete function satisfying the dynamics of (2). In this paper, we will study the algorithm (2) in more general settings.

Let $v^{i}(k)=\sum_{j \in N_{i}(k)} a_{j}^{i}(k) x^{j}(k)$ and $\varphi^{i}(k)=P_{X_{i}}\left[v^{i}(k)-\right.$ $\left.\alpha_{k} d_{i}(k)\right]-\left[v^{i}(k)-\alpha_{k} d_{i}(k)\right]$ for $i \in \mathcal{I}$. Here, $v^{i}(k)$ is the weighted average of agent $i$ 's neighbors' states at time $k$, playing a role in enabling all agents to reach a consensus, $-\alpha_{k} d_{i}(k)$ is a weighted negative subgradient, playing a role in minimizing the local function $f_{i}(x)$, and $\varphi^{i}(k)$ denotes a perturbation term at time $k$, yielding from the projection operation. The system (2) can be written in a compact form as $x(k+1)=v(k)-\gamma(k)+\varphi(k)=\mathcal{A}(k) x(k)-\gamma(k)+\varphi(k)$, where $x(k)=\left[x^{1}(k), \cdots, x^{n}(k)\right]^{T}, v(k)=\left[v^{1}(k), \cdots\right.$, $\left.v^{n}(k)\right]^{T}, \gamma(k)=\left[\alpha_{k} d_{1}(k), \cdots, \alpha_{k} d_{n}(k)\right]^{T}$, and $\varphi(k)=$ $\left[\varphi^{1}(k), \cdots, \varphi^{n}(k)\right]^{T}$. Let $\Psi(k, s)=\Pi_{j=s}^{k} \mathcal{A}(j)=$ $\mathcal{A}(k) \cdots \mathcal{A}(s)$ for all $k \geq s$ be the transition matrix of the system (2). Then, for all $k \geq s$, the system (2) can be written as

$$
\begin{aligned}
x^{i}(k+1) & =\sum_{j=1}^{n}[\Psi(k, s)]_{j}^{i} x^{j}(s)-\alpha_{k} d_{i}(k) \\
& -\sum_{r=s+1}^{k}\left(\sum_{j=1}^{n}[\Psi(k, r)]_{j}^{i} \alpha_{r-1} d_{j}(r-1)\right) \\
& +\sum_{r=s+1}^{k}\left(\sum_{j=1}^{n}[\Psi(k, r)]_{j}^{i} \varphi^{j}(r-1)\right)+\varphi^{i}(k) .
\end{aligned}
$$

Our main results for the case of fixed directed graphs are given in the following theorem.

Theorem 1 Suppose that the graph $\mathcal{G}$ is fixed, the adjacency matrix $\mathcal{A}$ is constant ${ }^{2}$, and strongly connected and Assumptions 1, 3(a), 5, and 6 hold. Using the algorith$\mathrm{m}$ (2), the multi-agent system reaches a common point $z^{*}$ in $X$ as $t \rightarrow+\infty$ and $z^{*}$ satisfies that $\sum_{i=1}^{n} q_{i} f_{i}\left(z^{*}\right)=$ $\min _{x \in X} \sum_{i=1}^{n} q_{i} f_{i}(x)$, where $q_{i}>0$ for all $i, \sum_{i=1}^{n} q_{i}=1$ and $\left[q_{1}, \cdots, q_{n}\right]$ is a left eigenvector of $\mathcal{A}$ associated with eigenvalue 1. Under an additional Assumption 3(b), $z^{*}$ satisfies that $\sum_{i=1}^{n} f_{i}\left(z^{*}\right)=\min _{x \in X} \sum_{i=1}^{n} f_{i}(x)$.

Remark 2 Theorem 1 essentially provides a complete analysis about the functionality of the algorithm (2) when the graph is unbalanced or balanced. Under a fixed graph, when consensus is achieved for any arbitrary initial condition with nonidentical constraints, the graph $\mathcal{G}$ should be strongly connected (see Remark 1). It can be concluded from Theorem 1 that distributed optimization is achieved if and only if the graph is strongly connected and balanced. When the graph is not balanced, the left eigenvector of $\mathcal{A}$ associated with the eigenvalue at 1 is not equal to $\frac{1^{T}}{n}$ and hence the final consensus value does not minimize the sum of the local functions $\sum_{i=1}^{n} f_{i}(x), x \in X$, but instead minimizes a weighted sum of the local functions. To make the algorithm solve the distributed optimization problem for a general unbalanced directed graph, future work could be towards calculating the left eigenvector associated with the eigenvalue at 1 of the matrix $\mathcal{A}$ in a distributed manner to compensate for the imbalance of the graph based on Theorem 1.

Our main results for the case of switching directed graphs are given in the following theorem.

Theorem 2 Suppose that Assumptions 1-6 hold. Using the algorithm (2), the multi-agent system reaches a common point $z^{*}$ in $X$ as $t \rightarrow+\infty$ and $z^{*}$ satisfies that $\sum_{i=1}^{n} f_{i}\left(z^{*}\right)=\min _{x \in X} \sum_{i=1}^{n} f_{i}(x)$.

Remark 3 In [2,4], all closed convex sets are assumed to be identical or the communication graphs are assumed to be fixed and fully connected. In [6], the communication graph is undirected and each possible communication link is functioning with a given probability. Thus, the communication graph by taking the expectation is essentially fixed and undirected. In contrast, Theorem 1 gives a complete analysis of

\footnotetext{
2 Hence Assumption 2 is automatically satisfied.
} 
the case of fixed directed graphs while Theorem 2 characterizes the case of switching directed graphs. In Theorem 2 , it is only required that the adjacency matrices be doubly stochastic and the union of the directed graphs is strongly connected among each time interval of a certain bounded length.

\subsection{Some necessary lemmas}

In this subsection, we give some lemmas that are used to prove the main theorems. Lemma 5 shows that each entry of the transition matrix $\Psi(k, s)$ is positive and nonvanishing over a certain time interval. Based on Lemma 5, Lemma 6 studies the convergence of each column of the transition matrix $\Psi(k, s)$ and shows that it converges to a certain vector with all equal positive elements exponentially. Lemma 7 establishes some properties of the nonzero vectors from the points in $X_{i}$ to their projections on the intersection set $X$ and shows that not all these vectors have a common direction when all $X_{i}$ are taken into account. Lemma 8 shows the consensus convergence property of the agents' states when the perturbation term $\varphi^{i}(k)$ and the step sizes tend to zero. Lemma 9 establishes the relationship between the distances from the agents to the intersection set $X$ at any two time steps and gives a bound for the magnitude of the perturbation term $\varphi^{i}(k)$ by reverse analysis. Lemma 10 proves the boundedness of the infinite sum of the product of the step sizes and the difference between $v^{i}(k)$ and the average of all agents' states over time.

Lemma 5 Under Assumptions 2, 3(a) and 4, there exists a constant $0<\mu<\frac{1}{n}$ such that $\left[\Psi\left(p_{2}, p_{1}\right)\right]_{j}^{i}>\mu$ for all $i, j$ and any $p_{2}-p_{1} \geq n M+2 M$.

Proof: Lemma 5 can be proved by a similar approach to the proof of Lemma 14(3) and hence omitted.

Lemma 6 Under Assumptions 2, 3(a) and 4,

(a) [2] Each transition matrix $\Psi(k, s)$ is a stochastic matrix for all $k \geq s$. Each column of $\Psi(k, s),[\Psi(k, s)]_{j}$, converges to a vector $\phi_{j}(s) \mathbf{1}$ as $k \rightarrow+\infty$, i.e., $\lim _{k \rightarrow+\infty}\left([\Psi(k, s)]_{j}-\phi_{j}(s) \mathbf{1}\right)=0$ for all $j$ where $\phi_{j}(s)>0$ and $\sum_{j=1}^{n} \phi_{j}(s)=1$.

(b) There is a constant $0<\mu<\frac{1}{n}$ such that $\|[\Psi(k, s)]_{j}^{i}-$ $\phi_{j}(s) \| \leq C_{0}(1-2 \mu)^{\frac{k-s}{M(n+2)}}$, where $C_{0}=(1-2 \mu)^{-1}$.

Proof: (b) The main idea of this proof is to calculate the difference of the maximum and minimum of each column of the transition matrix and show that the difference converges to zero exponentially, which implies that each entry of the transition matrix converges to its limit exponentially.

Note that $\Psi(k, s)$ is a stochastic matrix for all $k \geq s$ and $\Psi(k, s)$ can be written as $\Psi(k, s)=\Psi(k, \hat{k}) \Psi(\hat{k}-1, s)$ for all $k \geq \hat{k}>s \geq 0$. Each entry of $\Psi(k, s),[\Psi(k, s)]_{j}^{i}$, is a convex combination of the entries of the $j$ th column of $\Psi(\hat{k}-1, s)$. Then we have $\min _{i}\left\{[\Psi(\hat{k}-1, s)]_{j}^{i}\right\} \leq$ $\min _{i}\left\{[\Psi(k, s)]_{j}^{i}\right\} \leq \max _{i}\left\{[\Psi(k, s)]_{j}^{i}\right\} \leq \max _{i}\{[\Psi(\hat{k}-$ $\left.1, s)]_{j}^{i}\right\}$. Recall that $\phi_{j}(s)$ is the limit of $[\Psi(k, s)]_{j}^{i}$ as $k \rightarrow$ $+\infty$. We have $\min _{i}\left\{[\Psi(\hat{k}-1, s)]_{j}^{i}\right\} \leq \min _{i}\left\{[\Psi(k, s)]_{j}^{i}\right\} \leq$ $\phi_{j}(s) \leq \max _{i}\left\{[\Psi(k, s)]_{j}^{i}\right\} \leq \max _{i}\left\{[\Psi(\hat{k}-1, s)]_{j}^{i}\right\}$. It follows that $\max _{i}\left|[\Psi(k, s)]_{j}^{i}-\phi_{j}(s)\right| \leq \max _{i}\left\{[\Psi(k, s)]_{j}^{i}\right\}-$ $\min _{i}\left\{[\Psi(k, s)]_{j}^{i}\right\} \leq \max _{i}\left\{[\Psi(\hat{k}-1, s)]_{j}^{i}\right\}-\min _{i}\{[\Psi(\hat{k}-$ $\left.1, s)]_{j}^{i}\right\}$. Under Assumptions 2, 3(a) and 4, it follows from Lemma 5 that there exists a constant $0<$ $\mu<\frac{1}{n}$ such that $\left.[\Psi(s+(n+2) M), s)\right]_{j}^{i}>\mu$ for al$1 i, j \in \mathcal{I}$. Thus, $\left.\max _{i}\{[\Psi(s+(n+2) M), s)]_{j}^{i}\right\} \leq$ $\mu \min _{i}\left\{[\Psi(s, s)]_{j}^{i}\right\}+(1-\mu) \max _{i}\left\{[\Psi(s, s)]_{j}^{i}\right\} \quad$ and $\left.\min _{i}\{[\Psi(s+(n+2) M), s)]_{j}^{i}\right\} \geq(1-\mu) \min _{i}\left\{[\Psi(s, s)]_{j}^{i}\right\}+$ $\mu \max _{i}\left\{[\Psi(s, s)]_{j}^{i}\right\}$. It follows that

$$
\begin{aligned}
& \left.\max _{i}\{[\Psi(s+(n+2) M), s)]_{j}^{i}\right\} \\
- & \left.\min _{i}\{[\Psi(s+(n+2) M), s)]_{j}^{i}\right\} \\
\leq & (1-2 \mu)\left(\max _{i}\left\{[\Psi(s, s)]_{j}^{i}\right\}-\min _{i}\left\{[\Psi(s, s)]_{j}^{i}\right\}\right) \\
\leq & 1-2 \mu,
\end{aligned}
$$

where the second inequality holds by noting from the stochasticity and nonnegativity of the transition matrix $\Psi(s, s)$ that $\max _{i}\left\{[\Psi(s, s)]_{j}^{i}\right\}-\min _{i}\left\{[\Psi(s, s)]_{j}^{i}\right\} \leq 1$. Let $m_{1}=\left\lfloor\frac{k-s}{M n+2 M}\right\rfloor$ where $\left\lfloor\frac{k-s}{M n+2 M}\right\rfloor$ denotes the largest integer no larger than $\frac{k-s}{M n+2 M}$. By analogy, it can be proved that

$$
\begin{aligned}
& \max _{i}\left|[\Psi(k, s)]_{j}^{i}-\phi_{j}(s)\right| \\
\leq & \max _{i}\left\{[\Psi(k, s)]_{j}^{i}\right\}-\min _{i}\left\{[\Psi(k, s)]_{j}^{i}\right\} \\
\leq & \left.\max _{i}\left\{\left[\Psi\left(s+m_{1}(n+2) M\right), s\right)\right]_{j}^{i}\right\} \\
- & \left.\min _{i}\left\{\left[\Psi\left(s+m_{1}(n+2) M\right), s\right)\right]_{j}^{i}\right\} \\
\leq & (1-2 \mu)^{m_{1}} .
\end{aligned}
$$

Note that $m_{1} \geq \frac{k-s-M(n+2)}{M(n+2)}$ and $1-2 \mu<1$. Thus, we have $\left|[\Psi(k, s)]_{j}-\phi_{j}(s)\right| \leq(1-2 \mu)^{\frac{k-s-M(n+2)}{M(n+2)}}$.

Lemma 7 Suppose that Assumption 1 holds. Let $y^{i} \in \mathbb{R}^{m}$, $i \in \mathcal{I}$, be vectors such that $y^{i} \neq P_{X}\left(y^{i}\right)$.

(1) Let $a_{i} \geq 0, i \in \mathcal{I}$, be scalars such that $\sum_{i=1}^{n} a_{i}=$ 1. If $\left[y^{i}-P_{X}\left(y^{i}\right)\right] / /\left[y^{j}-P_{X}\left(y^{j}\right)\right]$ for all $i, j$, where // denotes that the two nonzero vectors have the same direction, $P_{X}\left(\sum_{i=1}^{n} a_{i} y^{i}\right)=\sum_{i=1}^{n} a_{i} P_{X}\left(y^{i}\right)$.

(2) If $y^{i} \in X_{i}, i \in \mathcal{I}$, there exist a pair of agents $i, j, i \neq j$ such that $\left\|y^{i}-P_{X}\left(y^{i}\right)+y^{j}-P_{X}\left(y^{j}\right)\right\|<\left\|y^{i}-P_{X}\left(y^{i}\right)\right\|+$ $\left\|y^{j}-P_{X}\left(y^{j}\right)\right\|$.

Proof: (1) Note that Assumption 1 ensures that $X$ is nonempty. From Lemma 1 , for any $\tilde{y} \in X$ and $\tilde{y} \neq$ 
$P_{X}\left(y^{i}(k)\right),\left\|y^{i}(k)-P_{X}\left(y^{i}(k)\right)\right\|^{2}+\left\|\tilde{y}-P_{X}\left(y^{i}(k)\right)\right\|^{2} \leq$ $\left\|y^{i}(k)-\tilde{y}\right\|^{2}$ for all $i$. This means that the angle between the vectors $y^{i}(k)-P_{X}\left(y^{i}(k)\right)$ and $\tilde{y}-P_{X}\left(y^{i}(k)\right)$ lies in $[\pi / 2, \pi]$ for all $i$. Here, the angles between two vectors are only considered in $[0, \pi]$. Since $P_{X}\left(y^{i}(k)\right) \in X$ for all $i$, it follows that the angle between the vectors $y^{i}(k)-P_{X}\left(y^{i}(k)\right)$ and $P_{X}\left(y^{j}(k)\right)-P_{X}\left(y^{i}(k)\right)$, denoted by $\beta_{i j}$, satisfies $\beta_{i j} \in[\pi / 2, \pi]$ for all $P_{X}\left(y^{j}(k)\right) \neq P_{X}\left(y^{i}(k)\right)$. Since $\left[y^{i}-P_{X}\left(y^{i}\right)\right] / /\left[y^{j}-P_{X}\left(y^{j}\right)\right]$ for all $i, j$, we have $\beta_{i j}+\beta_{j i}=\pi$ for all $P_{X}\left(y^{j}(k)\right) \neq P_{X}\left(y^{i}(k)\right)$. Thus, $\beta_{i j}=\beta_{j i}=\pi / 2$ for all $P_{X}\left(y^{j}(k)\right) \neq P_{X}\left(y^{i}(k)\right)$. This implies that for all $i$ the points $P_{X}\left(y^{i}(k)\right.$ lie on a common hyperplane, denoted by $\Gamma$, orthogonal to the vectors $y^{i}-P_{X}\left(y^{i}\right)$. Note that all $P_{X}\left(y^{i}(k)\right)$ lie on the boundary of $X$. Under Assumption 1, all the points of $X$ lie on one side of $\Gamma$ or on $\Gamma$, and all $y^{i}$ lie on the other side of $\Gamma$. Since $\sum_{i=1}^{n} a_{i} P_{X}\left(y^{i}\right)-\sum_{i=1}^{n} a_{i} y^{i}=$ $\sum_{i=1}^{n} a_{i}\left(P_{X}\left(y^{i}\right)-y^{i}\right)$ and $\sum_{i=1}^{n} a_{i} P_{X}\left(y^{i}\right) \in X$, $\sum_{i=1}^{n} a_{i} P_{X}\left(y^{i}\right)-\sum_{i=1}^{n} a_{i} y^{i}$ is orthogonal to $\Gamma$ and hence $P_{X}\left(\sum_{i=1}^{n} a_{i} y^{i}\right)=\sum_{i=1}^{n} a_{i} P_{X}\left(y^{i}\right)$.

(2) If this statement is not true, we have $\left[y^{i}-P_{X}\left(y^{i}\right)\right] / /\left[y^{j}-\right.$ $\left.P_{X}\left(y^{j}\right)\right]$ for all $i, j$. Thus, the vectors $y^{i}-P_{X}\left(y^{j}\right)$ and $y^{j}-P_{X}\left(y^{i}\right)$ must have an intersection point, denoted by $p_{i j}$, for $i \neq j$. Then we can select a convex combination of $P_{X}\left(y^{i}\right)$ and $P_{X}\left(y^{j}\right)$, denoted by $q_{i j}$, such that $\left[p_{i j}-\right.$ $\left.q_{i j}\right] / /\left[y^{i}-P_{X}\left(y^{i}\right)\right]$ for all $i, j$. Since $y^{i} \in X_{i}, y^{j} \in X_{j}$, $P_{X}\left(y^{i}\right) \in X$ and $P_{X}\left(y^{j}\right) \in X$, we have $p_{i j} \in X_{i} \bigcap X_{j}$. Since $p_{i j}$ is on the same side of $\Gamma$ as $y^{i}$ and $y^{j}$, we have $p_{i j} \notin X$. By analyzing the relationship between the vectors $p_{i j}-q_{i j}$ and $y^{j_{0}}-P_{X}\left(y^{j_{0}}\right)$ for $j_{0} \neq i$ and $j_{0} \neq j$ in a similar way, it can be proved that there exists one point $p_{i j j_{0}}$ such that $p_{i j j_{0}} \in X_{i} \bigcap X_{j} \cap X_{j_{0}}$ and $p_{i j j_{0}} \notin X$. By analogy, it can be proved that there exists one point $p$ such that $p \in$ $\bigcap_{i=1}^{n} X_{i}=X$ and $p \notin X$, which is a contradiction.

Lemma 8 Suppose that $\left\|\varphi^{i}(k)\right\|<\epsilon$ and $0<\alpha_{k}<\epsilon / L$ for a constant $\epsilon>0$, all $k>s$ and all $i$, where $s$ is the initial time of the system (3). Under Assumptions 2, 3(a), 4 and 5, when $\epsilon$ is sufficiently small and $k-s$ is sufficiently large, for any $\omega>0$, the inequality $\left\|x^{i}(k)-x^{j}(k)\right\|<\omega$ holds for all $i, j$.
Proof: Note that $\sum_{i=1}^{n} \phi_{j}(r)=n \phi_{j}(r)$ and hence $-\phi_{j}(r)+$ $\frac{1}{n} \sum_{i=1}^{n} \phi_{j}(r)=0$ for all $r$. From (3),

$$
\begin{aligned}
& \left\|x^{i}(k)-x^{j}(k)\right\| \\
\leq & \left\|x^{i}(k)-\frac{1}{n} \sum_{i=1}^{n} x^{i}(k)\right\|+\left\|x^{j}(k)-\frac{1}{n} \sum_{i=1}^{n} x^{i}(k)\right\| \\
\leq & 2 \max _{i}\left\|x^{i}(k)-\frac{1}{n} \sum_{i=1}^{n} x^{i}(k)\right\| \\
= & 2 \max _{i} \| \sum_{j=1}^{n}\left[\left([\Psi(k-1, s)]_{j}^{i}-\phi_{j}(s)\right)\right. \\
- & \left.\frac{1}{n} \sum_{i=1}^{n}\left([\Psi(k-1, s)]_{j}^{i}-\phi_{j}(s)\right)\right] x^{j}(s) \\
- & \sum_{r=s+1}^{k-1}\left\{\sum _ { j = 1 } ^ { n } \left[\left([\Psi(k-1, r)]_{j}^{i}-\phi_{j}(r)\right)\right.\right. \\
- & \left.\left.\frac{1}{n} \sum_{i=1}^{n}\left([\Psi(k-1, r)]_{j}^{i}-\phi_{j}(r)\right)\right] \alpha_{r-1} d_{j}(r-1)\right\} \\
- & \left(\alpha_{k-1} d_{i}(k-1)-\frac{1}{n} \sum_{j=1}^{n} \alpha_{k-1} d_{j}(k-1)\right) \\
+ & \sum_{r=s+1}^{k-1}\left\{\sum _ { j = 1 } ^ { n } \left[\left([\Psi(k-1, r)]_{j}^{i}-\phi_{j}(r)\right)\right.\right. \\
- & \left.\left.\frac{1}{n} \sum_{i=1}^{n}\left([\Psi(k-1, r)]_{j}^{i}-\phi_{j}(r)\right)\right] \varphi^{j}(r-1)\right\} \\
+ & \left(\varphi^{i}(k-1)-\frac{1}{n} \sum_{j=1}^{n} \varphi^{j}(k-1)\right) \|,
\end{aligned}
$$

where the limit $\phi_{j}$ is defined in Lemma 6. Under Assumptions 2, 3(a), 4 and 5, Lemmas 4 and 6 hold. Since $\left\|\varphi^{i}(k)\right\|<$ $\epsilon$ and $0<\alpha_{k}<\epsilon / L$ for all $k>s$ and all $i$, it follows that for all $i$ and all $k \geq s+1$ there exist two constants $C_{0}>0$ and $0<\mu<\frac{1}{n}$ such that

$$
\begin{aligned}
& \left\|x^{i}(k)-x^{j}(k)\right\| \\
\leq & 4 n C_{0}(1-2 \mu)^{\frac{k-s-1}{M(n+2)}} \max _{j}\left\|x^{j}(s)\right\| \\
+ & \sum_{r=s+1}^{k-1} 8 n C_{0}(1-2 \mu)^{\frac{k-r-1}{M(n+2)}} \epsilon+8 \epsilon \\
\leq & 4 n C_{0}(1-2 \mu)^{\frac{k-s-1}{M(n+2)}} \max _{j}\left\|x^{j}(s)\right\| \\
+ & 8 n C_{0} \frac{1}{1-(1-2 \mu)^{\frac{1}{M(n+2)}}} \epsilon+8 \epsilon .
\end{aligned}
$$

Note that $n>1$ and hence $0<1-2 \mu<1$. Thus, when $\epsilon$ is sufficiently small and $k-s$ is sufficiently large, the inequality $\left\|x^{i}(k)-x^{j}(k)\right\|<\omega$ holds for any $\omega>0$ and all $i, j$.

Lemma 9 Under Assumptions 1, 2, 3(a), 4 and 5, the following statements hold.

(a) For all $i$,

$$
\begin{aligned}
& \sqrt{\left\|x^{i}(k+1)-P_{X}\left(x^{i}(k+1)\right)\right\|^{2}+\left\|\varphi^{i}(k)\right\|^{2}} \\
\leq & \sum_{j \in N_{i}(k)} a_{j}^{i}(k)\left\|x^{j}(k)-P_{X}\left(x^{j}(k)\right)\right\|+2 \alpha_{k} L .
\end{aligned}
$$

(b) There exists a constant $0 \leq c_{\mu}<1$ for any $k>s$ such that

$$
\begin{aligned}
& \max _{i}\left\|x^{i}(k)-P_{X}\left(x^{i}(k)\right)\right\| \\
\leq & 2 \sum_{r=s}^{k-1} c_{\mu}^{-1} c_{\mu}^{\frac{k-r-1}{n M+4 M}} \alpha_{r} L \\
+ & c_{\mu}^{-1} c_{\mu}^{\frac{k-s}{n M+4 M}} \max _{i}\left\|x^{i}(s)-P_{X}\left(x^{i}(s)\right)\right\| .
\end{aligned}
$$


(c) For all $i$,

$$
\begin{aligned}
& \left\|\varphi^{i}(k)\right\|^{2} \leq\left[\sum_{j \in N_{i}(k)} a_{j}^{i}(k)\left\|x^{j}(k)-P_{X}\left(x^{j}(k)\right)\right\|\right]^{2} \\
+ & 4 \alpha_{k} L\left[\sum_{j \in N_{i}(k)} a_{j}^{i}(k)\left\|x^{j}(k)-P_{X}\left(x^{j}(k)\right)\right\|\right] \\
+ & 4 \alpha_{k}^{2} L^{2}-\left\|x^{i}(k+1)-P_{X}\left(x^{i}(k+1)\right)\right\|^{2} .
\end{aligned}
$$

Proof: (a) Under Assumption 1, $X$ is nonempty. Note that $\left\|x-P_{X}(x)\right\|^{2} \leq\|x-y\|^{2}$ for all $x \in \mathbb{R}^{m}$ and all $y \in X$ and $P_{X}\left(v^{i}(k)-\alpha_{k} d_{i}(k)\right) \in X_{i}$. It follows from Lemma 1 that for all $i$

$$
\begin{aligned}
& \left\|x^{i}(k+1)-P_{X}\left(x^{i}(k+1)\right)\right\|^{2} \\
= & \| P_{X_{i}}\left(v^{i}(k)-\alpha_{k} d_{i}(k)\right) \\
- & P_{X}\left(P_{X_{i}}\left(v^{i}(k)-\alpha_{k} d_{i}(k)\right)\right) \|^{2} \\
\leq & \left\|P_{X_{i}}\left(v^{i}(k)-\alpha_{k} d_{i}(k)\right)-P_{X}\left(v^{i}(k)-\alpha_{k} d_{i}(k)\right)\right\|^{2} \\
\leq & \left\|v^{i}(k)-\alpha_{k} d_{i}(k)-P_{X}\left(v^{i}(k)-\alpha_{k} d_{i}(k)\right)\right\|^{2} \\
- & \left\|\varphi^{i}(k)\right\|^{2} .
\end{aligned}
$$

Using Lemma 2, we have for all $i$

$$
\begin{aligned}
& \left\|v^{i}(k)-\alpha_{k} d_{i}(k)-P_{X}\left(v^{i}(k)-\alpha_{k} d_{i}(k)\right)\right\| \\
= & \| v^{i}(k)-P_{X}\left(v^{i}(k)\right)-\alpha_{k} d_{i}(k)+P_{X}\left(v^{i}(k)\right) \\
- & P_{X}\left(v^{i}(k)-\alpha_{k} d_{i}(k)\right) \| \\
\leq & \left\|v^{i}(k)-P_{X}\left(v^{i}(k)\right)\right\|+\| \alpha_{k} d_{i}(k) \\
- & P_{X}\left(v^{i}(k)\right)+P_{X}\left(v^{i}(k)-\alpha_{k} d_{i}(k)\right) \| \\
\leq & \sum_{j \in N_{i}(k)} a_{j}^{i}(k)\left\|x^{j}(k)-P_{X}\left(x^{j}(k)\right)\right\|+\| \alpha_{k} d_{i}(k) \\
- & P_{X}\left(v^{i}(k)\right)+P_{X}\left(v^{i}(k)-\alpha_{k} d_{i}(k)\right) \| .
\end{aligned}
$$

Under Assumptions 1 and 5, it follows from Lemmas 1 and 4 that $\left\|P_{X}\left(v^{i}(k)\right)-P_{X}\left(v^{i}(k)-\alpha_{k} d_{i}(k)\right)\right\| \leq\left\|\alpha_{k} d_{i}(k)\right\| \leq$ $\alpha_{k} L$. Hence (4) follows from (7) and (8) straightforwardly.

(b) To prove this statement, the key step of our approach is to prove that there exists a constant $0 \leq c_{\mu}<1$ for any $k-s=n M+4 M$ such that $\max _{i}\left\|x^{i}(k)-P_{X}\left(x^{i}(k)\right)\right\| \leq$ $c_{\mu} \max _{i}\left\|x^{i}(s)-P_{X}\left(x^{i}(s)\right)\right\|+2 \sum_{r=s}^{k-1} \alpha_{r} L$. Let $m$ be the smallest integer such that $k_{m} \geq s$. First, we consider the case where $\left\|x^{i_{0}}(r)-P_{X}\left(x^{i_{0}}(r)\right)\right\|=0$ for some $i_{0}$ and some $s \leq r \leq k_{m+1}$. Note that $k-r \geq n M+2 M$ for any $k-s=n M+4 M$. Under Assumption 2, 3(a) and 4 , it follows from Lemma 5 that there exists a constant $0<\mu<\frac{1}{n}$ such that $[\Psi(k, r)]_{j}^{i}>\mu$ for all $i, j$ and any $k-s=n M+4 M$. Thus, from (4), using the stochasticity of the matrices $\Psi(\cdot)$, we have

$$
\begin{aligned}
& \max _{i}\left\|x^{i}(k)-P_{X}\left(x^{i}(k)\right)\right\| \\
\leq & \max _{i}\left\{\sum_{j=1, j \neq i_{0}}^{n}[\Psi(k-1, r)]_{j}^{i}\left\|x^{j}(r)-P_{X}\left(x^{j}(r)\right)\right\|\right. \\
+ & {\left.[\Psi(k-1, r)]_{i_{0}}^{i}\left\|x^{i_{0}}(r)-P_{X}\left(x^{i_{0}}(r)\right)\right\|\right\}+2 \sum_{h=r}^{k-1} \alpha_{h} L } \\
\leq & \max _{i}\left\{\sum_{j=1, j \neq i_{0}}^{n}[\Psi(k-1, r)]_{j}^{i}\left\|x^{j}(r)-P_{X}\left(x^{j}(r)\right)\right\|\right\} \\
+ & 2 \sum_{h=r}^{k-1} \alpha_{h} L \\
\leq & c_{k s} \max _{i}\left\|x^{i}(r)-P_{X}\left(x^{i}(r)\right)\right\|+2 \sum_{h=r}^{k-1} \alpha_{h} L
\end{aligned}
$$

where $1>c_{k s} \geq 1-\mu>1-[\Psi(k-1, r)]_{i_{0}}^{i}=$ $\sum_{j=1, j \neq i_{0}}^{n}[\Psi(k-1, r)]_{j}^{i}$. Similarly, it can be obtained that $\max _{i}\left\|x^{i}(r)-P_{X}\left(x^{i}(r)\right) \leq \max _{i}\right\| x^{i}(s)-P_{X}\left(x^{i}(s)\right) \|+$ $2 \sum_{h=s}^{r-1} \alpha_{h} L$. Therefore, $\max _{i}\left\|x^{i}(k)-P_{X}\left(x^{i}(k)\right)\right\| \leq$ $c_{k s} \max _{i}\left\|x^{i}(s)-P_{X}\left(x^{i}(s)\right)\right\|+2 \sum_{h=s}^{k-1} \alpha_{h} L$.

Second, we consider the case where $\left\|x^{i}(k)-P_{X}\left(x^{i}(k)\right)\right\| \neq$ 0 for all $i$ and all $s \leq k \leq k_{m+1}$. Under Assumption 1, Lemma 7 holds. According to the proof of Lemma 2, using Lemma 7(1), for all $i$ and all $s \leq k \leq k_{m+1}$, the third equality of (8) implies that $v^{i}(k)-\bar{P}_{X}\left(v^{i}(k)\right) \neq$ 0 and $\left[v^{i}(k)-P_{X}\left(v^{i}(k)\right)\right] / /\left[x^{j}(k)-P_{X}\left(x^{j}(k)\right)\right]$ for al$1 j \in N_{i}(k)$, where // has been defined in Lemma 7 . Moreover, for all $i$ and all $s \leq k \leq k_{m+1}$, if the second equality of (8) further holds and $\left\|\varphi^{i}(k)\right\|=0$, i.e., $x^{i}(k+1)=v^{i}(k)-\alpha_{k} d_{i}(k)$, we have $\left[v^{i}(k)-\alpha_{k} d_{i}(k)-\right.$ $\left.P_{X}\left(v^{i}(k)-\alpha_{k} d_{i}(k)\right)\right] / /\left[v^{i}(k)-P_{X}\left(v^{i}(k)\right)\right]$, i.e., $\left[x^{i}(k+\right.$ 1) $-P_{X}\left(x^{i}(k+1)\right] / /\left[v^{i}(k)-P_{X}\left(v^{i}(k)\right)\right]$. As a result, when the equalities of (8) all hold and $\left\|\varphi^{i}(k)\right\|=0$ for al$1 i$ and all $s \leq k \leq k_{m+1}$, under Assumption 4, it is not hard to see that $\left[x^{i}\left(k_{m+1}\right)-P_{X}\left(x^{i}\left(k_{m+1}\right)\right)\right] / /\left[x^{j}\left(k_{m+1}\right)-\right.$ $\left.P_{X}\left(x^{j}\left(k_{m+1}\right)\right)\right]$ for all $i, j$. This contradicts with Lemma $7(2)$. Thus, we have that one of the equalities of (8) does not hold or $\left\|\varphi^{i_{0}}(r)\right\| \neq 0$ for some $i_{0}$ and some $s \leq r \leq k_{m+1}$, which both imply from (4) that $\| x^{i_{0}}(r+1)-P_{X}\left(x^{i_{0}}(r+\right.$ 1)) $\left\|\leq c_{r} \sum_{j \in N_{i_{0}}(r)} a_{j}^{i_{0}}(r)\right\| x^{j}(r)-P_{X}\left(x^{j}(r)\right) \|+2 \alpha_{r} L$ for some constant $0 \leq c_{r}<1$. Take $k-s=n M+4 M$. Clearly, $k-r>n M+2 M$. Under Assumptions 2, 3(a) and 4, it follows from Lemma 5 that $\left\|x^{i}(k)-P_{X}\left(x^{i}(k)\right)\right\| \leq$ $c_{k s} \sum_{j=1}^{n}[\Psi(k-1, r)]_{j}^{i}\left\|x^{j}(r)-P_{X}\left(x^{j}(r)\right)\right\|+2 \sum_{h=r}^{k-1} \alpha_{h} L$ for all $i$ and some constant $0 \leq c_{k s}<1$. Thus, similar to the analysis of the first case, we have $\left\|x^{i}(k)-P_{X}\left(x^{i}(k)\right)\right\| \leq$ $c_{k s} \sum_{j=1}^{n}[\Psi(k-1, s)]_{j}^{i}\left\|x^{j}(s)-P_{X}\left(x^{j}(s)\right)\right\|+2 \sum_{r=s}^{k-1} \alpha_{r} L$ for all $i$ and some constant $0 \leq c_{k s}<1$.

Note from Assumption 5 that all $X_{i}$ and $X$ are bounded closed sets and $\eta \leq a_{i}^{j}(k) \leq 1$ for all $k$ and the parameter $c_{k s}$ can be regarded as a continuous function of all $x^{i}(s)$ and all $a_{i}^{j}(r)$ for $s \leq r \leq k$ and $k-s=n M+4 M$. According to the property of continuous functions on bounded closed sets, from the analysis of the above two cases, there must exist a constant $0 \leq c_{\mu}<1$ for any $k-s=n M+4 M$ such that $\max _{i}\left\|x^{i}(k)-P_{X}\left(x^{i}(k)\right)\right\| \leq c_{\mu} \max _{i} \| x^{i}(s)-$ $P_{X}\left(x^{i}(s)\right) \|+2 \sum_{r=s}^{k-1} \alpha_{r} L$. Then similar to the latter part 
of the proof of Lemma 6 , for any $k>s, \max _{i} \| x^{i}(k)-$ $P_{X}\left(x^{i}(k)\right)\left\|\leq c_{\mu}^{-1} c_{\mu}^{\frac{k-s}{n M+4 M}} \max _{i}\right\| x^{i}(s)-P_{X}\left(x^{i}(s)\right) \|+$ $2 \sum_{r=s}^{k-1} c_{\mu}^{-1} c_{\mu}^{\frac{k-r-1}{n M+4 M}} \alpha_{r} L$.

(c) From (7) and (8), for all $i$

$$
\begin{aligned}
& \left\|x^{i}(k+1)-P_{X}\left(x^{i}(k+1)\right)\right\|^{2} \\
\leq & \left\|v^{i}(k)-\alpha_{k} d_{i}(k)-P_{X}\left(v^{i}(k)-\alpha_{k} d_{i}(k)\right)\right\|^{2} \\
- & \left\|\varphi^{i}(k)\right\|^{2} \\
\leq & \left(\left\|v^{i}(k)-P_{X}\left(v^{i}(k)\right)\right\|+2 \alpha_{k} L\right)^{2}-\left\|\varphi^{i}(k)\right\|^{2} \\
\leq & {\left[\sum_{j \in N_{i}(k)} a_{j}^{i}(k)\left\|x^{j}(k)-P_{X}\left(x^{j}(k)\right)\right\|\right]^{2}+4 \alpha_{k}^{2} L^{2} } \\
+ & 4 \alpha_{k} L\left[\sum_{j \in N_{i}(k)} a_{j}^{i}(k)\left\|x^{j}(k)-P_{X}\left(x^{j}(k)\right)\right\|\right] \\
- & \left\|\varphi^{i}(k)\right\|^{2} .
\end{aligned}
$$

Thus, (6) holds.

Lemma 10 Define the average of all $x^{i}(k)$ as $x_{\text {ave }}(k)=$ $\frac{1}{n} \sum_{i=1}^{n} x^{i}(k)$. Under Assumptions 1, 2, 3(a), 4, 5 and 6, $\sum_{k=0}^{+\infty} \alpha_{k}\left\|v^{i}(k)-P_{X}\left(x_{\text {ave }}(k)\right)\right\|<+\infty$ for all $i$.

Proof: Under Assumptions 1, 2, 3(a), 4 and 5, Lemma 9 holds. From Lemma 9(a), $\max _{i}\left\|\varphi^{i}(k)\right\| \leq \max _{i} \| x^{i}(k)-$ $P_{X}\left(x^{i}(k)\right) \|+2 \alpha_{k} L$. By a similar way to the proof of Lemma 8 and applying Lemma 9(b), there exist two constants $C_{0}>$ 0 and $0<\mu<\frac{1}{n}$ such that

$$
\begin{aligned}
& \max _{i}\left\|x^{i}(k)-x_{\text {ave }}(k)\right\| \\
< & 2 n C_{0}(1-2 \mu)^{\frac{k-1}{M n+2 M}} \max _{j}\left\|x^{j}(0)\right\| \\
+ & \sum_{r=1}^{k-1} 4 n C_{0}(1-2 \mu)^{\frac{k-1-r}{M n+2 M}} \alpha_{r-1} L+4 \alpha_{k-1} L \\
+ & \sum_{r=1}^{k-1} 2 n C_{0}(1-2 \mu)^{\frac{k-1-r}{M n+2 M}}\left[c_{\mu}^{-1} c_{\mu}^{\frac{r-1}{n M+4 M}}\right. \\
\times & \max _{i}\left\|x^{i}(0)-P_{X}\left(x^{i}(0)\right)\right\| \\
+ & \left.2 \sum_{h=0}^{r-2} c_{\mu}^{-1} c_{\mu}^{\frac{r-h-2}{n M+4 M}} \alpha_{h} L\right] \\
+ & 2 c_{\mu}^{-1} c_{\mu}^{\frac{k_{-1}}{n M+4 M}} \max \left\|x^{i}(0)-P_{X}\left(x^{i}(0)\right)\right\| \\
+ & 2 \sum_{r=0}^{k-2} c_{\mu}^{-1} c_{\mu}^{\frac{k-r-2}{n M+4 M}} \alpha_{r} L .
\end{aligned}
$$

Under Assumption 1, Lemma 3 holds. Together with Assumption 5, we have $\left\|x_{\text {ave }}(k)-P_{X}\left(x_{\text {ave }}(k)\right)\right\| \leq$ $\frac{2 H}{\delta} \sum_{i=1}^{n}\left\|x_{\text {ave }}(k)-P_{X_{i}}\left(x_{\text {ave }}(k)\right)\right\|$. Note that $\| x_{\text {ave }}(k)-$ $P_{X_{i}}\left(x_{\text {ave }}(k)\right)\|\leq\| x_{\text {ave }}(k)-x^{i}(k) \|$ from the definition of the projection operator. It follows that

$$
\begin{aligned}
& \left\|v^{i}(k)-P_{X}\left(x_{\text {ave }}(k)\right)\right\| \\
\leq & \left\|v^{i}(k)-x_{\text {ave }}(k)\right\|+\left\|x_{\text {ave }}(k)-P_{X}\left(x_{\text {ave }}(k)\right)\right\| \\
\leq & \max _{i}\left\|x^{i}(k)-x_{\text {ave }}(k)\right\|+\frac{2 H}{\delta} \sum_{i=1}^{n}\left\|x_{\text {ave }}(k)-x^{i}(k)\right\| \\
\leq & \left(1+\frac{2 n H}{\delta}\right) \max _{i}\left\|x^{i}(k)-x_{\text {ave }}(k)\right\| .
\end{aligned}
$$

Under Assumption 6, $\lim _{k \rightarrow+\infty} \alpha_{k}=0$. Hence, $\alpha_{k}$ are upper bounded for all $k$. Note that $0<1-2 \mu<1$. From (9), there must exist constants $c_{1}>0$ and $0<c_{2}<1$ such that

$$
\begin{aligned}
& \sum_{k=0}^{+\infty} \alpha_{k}\left\|v^{i}(k)-P_{X}\left(x_{\mathrm{ave}}(k)\right)\right\| \\
\leq & \sum_{k=0}^{+\infty} c_{1} c_{2}^{k}+\sum_{k=0}^{+\infty} \alpha_{k}\left[\sum_{r=1}^{k-1} c_{1} c_{2}^{k-1-r} \alpha_{r-1}\right. \\
+ & \left.c_{1} \alpha_{k-1}\right]+\sum_{k=0}^{+\infty} \sum_{r=1}^{k-1}\left[c_{1} c_{2}^{k}+\alpha_{k} \sum_{h=0}^{r-2} c_{1} c_{2}^{k-h-2} \alpha_{h}\right] \\
+ & \sum_{k=0}^{+\infty}\left[c_{1} c_{2}^{k}+\alpha_{k} \sum_{r=1}^{k-1} c_{1} c_{2}^{k-r-1} \alpha_{r-1}\right] \\
\leq & \sum_{k=0}^{+\infty}(k+1) c_{1} c_{2}^{k} \\
+ & \sum_{k=0}^{+\infty}\left[\sum_{r=1}^{k-1} c_{1} c_{2}^{k-1-r}\left(\alpha_{r-1}^{2}+\alpha_{k}^{2}\right)+c_{1}\left(\alpha_{k-1}^{2}+\alpha_{k}^{2}\right)\right] \\
+ & \sum_{k=0}^{+\infty} \sum_{r=1}^{k-1} \sum_{h=0}^{r-2} c_{1} c_{2}^{k-h-2}\left(\alpha_{h}^{2}+\alpha_{k}^{2}\right)
\end{aligned}
$$

where the second inequality is due to the inequality $2 \alpha_{i} \alpha_{j} \leq \alpha_{i}^{2}+\alpha_{j}^{2}$ for any $i, j$. After some calculation$\mathrm{s}$, we have $\sum_{k=0}^{+\infty}(k+1) c_{1} c_{2}^{k} \leq c_{1} /\left(1-c_{2}\right)^{2}<+\infty$, $\sum_{k=0}^{+\infty} \sum_{r=1}^{k-1} c_{1} c_{2}^{k-1-r}\left(\alpha_{r-1}^{2}+\alpha_{k}^{2}\right) \leq 2 \sum_{k=0}^{+\infty} c_{1} /(1-$ $\left.c_{2}\right) \alpha_{k}^{2}<+\infty$, and $\sum_{k=0}^{+\infty} \sum_{r=1}^{k-1} \sum_{h=0}^{r-2} c_{1} c_{2}^{k-h-2}\left(\alpha_{h}^{2}+\alpha_{k}^{2}\right)$ $\leq 2 c_{1} /\left(1-c_{2}\right)^{2} \sum_{i=1}^{+\infty} \alpha_{i}^{2}<+\infty$. From Assumption 6 and (10), we have $\sum_{k=0}^{+\infty} \alpha_{k}\left\|v^{i}(k)-P_{X}\left(x_{\text {ave }}(k)\right)\right\|<+\infty$.

\subsection{Proofs of Theorems 1 and 2}

To prove Theorem 1, our analysis is given mainly in three steps: (1) use Lemmas 8 and 9 to prove by reverse analysis with a contradiction argument that the distances from all agents to the intersection set $X$ and hence the perturbation terms $\varphi^{i}(k)$ approach zero as $k \rightarrow+\infty$ (Lemmas 11 and 12); (2) prove that $x^{i}(k)$ and $v^{i}(k)$ converge to the average value of all $x^{i}(k)$ (Proposition 1); (3) use the projection property and Lemmas 6, 10 and 13 and Proposition 1 to estimate the distance from each $x^{i}(k)$ to the point, denoted by $\hat{z}^{*}$, that optimizes a weighted sum of the local objective functions so as to show the convergence of each $x^{i}(k)$ to $\hat{z}^{*}$.

Lemma 11 Under Assumptions 1, 2, 3(a), 4, 5 and 6, for all $i, \lim _{k \rightarrow+\infty}\left\|x^{i}(k)-P_{X}\left(x^{i}(k)\right)\right\|=0$.

Proof: Under Assumptions 5 and $6,\left\|x^{i}(k)-P_{X}\left(x^{i}(k)\right)\right\|$ is bounded and $\lim _{k \rightarrow+\infty} \alpha_{k}=0$. If this lemma does not hold, then the superior limit of the sequence $\left\{\max _{i} \| x^{i}(k)-\right.$ $\left.P_{X}\left(x^{i}(k)\right) \|\right\}$ is larger than zero, denoted by $D_{1}>0$. Then there must exist integers $i_{0}, l_{P}$ and $\bar{k}$ for any $\epsilon>0$ and any $N_{P}>(n+2) M+1$ such that $D_{1}-\epsilon<\left\|x^{i_{0}}(\bar{k})-P_{X}\left(x^{i_{0}}(\bar{k})\right)\right\|<D_{1}+\epsilon, \bar{k}-l_{P}>N_{P}$, $\left\|x^{i}(k)-P_{X}\left(x^{i}(k)\right)\right\|<D_{1}+\epsilon$ and $0<\alpha_{k}<\epsilon$ for all $i$ and all $k>l_{P}$.

Denote $Z_{P}(k)=\left[\left\|x^{1}(k)-P_{X}\left(x^{1}(k)\right)\right\|, \cdots, \| x^{n}(k)-\right.$ $\left.P_{X}\left(x^{n}(k)\right) \|\right]^{T}$. Under Assumptions 1, 2, 3(a), 4 and 5, Lemma 9 holds. Then from (4) we have $Z_{P}(k+1) \leq$ $\Psi(k, k) Z_{P}(k)+2 \alpha_{k} L \mathbf{1}_{n} \leq \Psi(k, k) Z_{P}(k)+2 \epsilon L \mathbf{1}_{n}$ for all 
$k>l_{P}$ and hence

$$
\begin{aligned}
& Z_{P}(\bar{k}) \leq \Psi(\bar{k}-1, k) Z_{P}(k) \\
& +\sum_{r=k+1}^{\bar{k}-1} \Psi(\bar{k}-1, r) \times\left(2 \epsilon L \mathbf{1}_{n}\right)+2 \epsilon L \mathbf{1}_{n} \\
& =\Psi(\bar{k}-1, k) Z_{P}(k)+2(\bar{k}-k) \epsilon L \mathbf{1}_{n}
\end{aligned}
$$

for all $l_{P}<k<\bar{k}$ in view of the stochasticity of $\Psi(\bar{k}-1, k)$. Further, since $Z_{P}^{i}(k)<D_{1}+\epsilon$ for all $k>l_{P}$, it follows that

$$
\begin{aligned}
Z_{P}^{i_{0}}(\bar{k}) & \leq \sum_{j=1, j \neq i}^{n} \Psi_{j}^{i_{0}}(\bar{k}-1, k) Z_{P}^{j}(k) \\
& +2(\bar{k}-k) \epsilon L+\Psi_{i}^{i_{0}}(\bar{k}-1, k) Z_{P}^{i}(k) \\
& \leq\left(1-\Psi_{i}^{i_{0}}(\bar{k}-1, k)\right)\left(D_{1}+\epsilon\right) \\
& +2(\bar{k}-k) \epsilon L+\Psi_{i}^{i_{0}}(\bar{k}-1, k) Z_{P}^{i}(k)
\end{aligned}
$$

for all $i$ and all $l_{P}<k<\bar{k}$. Under Assumptions 2, 3(a) and 4 , it follows from Lemma 5 that there exists a positive number $\mu>0$ such that $\Psi_{i}^{i_{0}}(\bar{k}-1, k)>\mu$ for all $l_{P}<$ $k<\bar{k}-(n+2) M-1$ and all $i$. Here, the solution set of the inequality $l_{P}<k<\bar{k}-(n+2) M-1$ is nonempty since $\bar{k}-l_{P}>N_{P}>(n+2) M+1$. Note that $D_{1}-\epsilon<$ $\left\|x^{i_{0}}(\bar{k})-P_{X}\left(x^{i_{0}}(\bar{k})\right)\right\|$. It follows that

$$
\begin{aligned}
Z_{P}^{i}(k) & \geq\left[D_{1}-\epsilon-\left(1-\Psi_{i}^{i_{0}}(\bar{k}-1, k)\right)\left(D_{1}+\epsilon\right)\right. \\
& -2(\bar{k}-k) \epsilon L] / \Psi_{i}^{i_{0}}(\bar{k}-1, k) \\
& \geq D_{1}-[2 \epsilon+2(\bar{k}-k) \epsilon L] / \mu+\epsilon
\end{aligned}
$$

for all $l_{P}<k<\bar{k}-(n+2) M-1$ and all $i$. From (6) and (13), it follows that

$$
\begin{aligned}
& \left\|\varphi^{i}(k)\right\|^{2} \\
\leq & {\left[\sum_{j \in N_{i}(k)} a_{j}^{i}(k) Z_{P}^{j}(k)\right]^{2} } \\
+ & 4 \alpha_{k} L\left[\sum_{j \in N_{i}(k)} a_{j}^{i}(k) Z_{P}^{j}(k)\right]+4 \alpha_{k}^{2} L^{2}-Z_{P}^{i}(k+1)^{2} \\
\leq & \left(D_{1}+\epsilon\right)^{2}+4 \epsilon L\left(D_{1}+\epsilon\right)+4 \epsilon^{2} L^{2} \\
- & \left\{D_{1}-[2 \epsilon+2(\bar{k}-k-1) \epsilon L] / \mu+\epsilon\right\}^{2} \\
\leq & \{4 \epsilon L+[4 \epsilon+4(\bar{k}-k-1) \epsilon L] / \mu\}\left(D_{1}+\epsilon\right)+4 \epsilon^{2} L^{2} \\
- & {[2 \epsilon+2(\bar{k}-k-1) \epsilon L]^{2} / \mu^{2} }
\end{aligned}
$$

for all $l_{P}<k<\bar{k}-(n+2) M-2$ and all $i$, where the second inequality holds since $\sum_{j \in N_{i}(k)} a_{j}^{i}(k)=1$.

Because $\epsilon$ and $N_{P}$ can be arbitrarily chosen, let $\epsilon$ be sufficiently small and $N_{P}$ be sufficiently large. Under Assumptions 2, 3(a), 4 and 5, it follows from Lemma 8 that, for any $\omega>0$, the inequality $\left\|x^{i}(k)-x^{j}(k)\right\|<\omega$ holds for all $i, j$ and all $l_{P}+N_{P}-(n+2) M-2<k<\bar{k}-(n+2) M-2$.

On the other hand, consider the set $\bar{X}_{i}(k)=\left\{x \in X_{i} \mid\right.$ $D_{1}-[2 \epsilon+2(\bar{k}-k-1) \epsilon L] / \mu+\epsilon<\left\|x-P_{X}(x)\right\|<$ $\left.D_{1}+\epsilon\right\}$ for all $i$ and all $l_{P}+N_{P}-(n+2) M-2<k<$ $\bar{k}-(n+2) M-2$. Clearly, when $\epsilon$ is sufficiently small, $\left\|x-P_{X}(x)\right\|>D_{1}-[2 \epsilon+2(\bar{k}-k-1) \epsilon L] / \mu+\epsilon>0$ for all $x \in \bar{X}_{i}$, i.e., $\bar{X}_{i}(k) \cap X=\emptyset$, for all $i$. It follows that $\bar{\omega}=$ $\min _{i} \max _{j, x \in \bar{X}_{i}(k)}\left\|x-P_{X_{j}}(x)\right\| \neq 0$ for sufficiently small $\epsilon$. Note that $\max _{i, j}\left\|x^{i}(k)-x^{j}(k)\right\| \geq \max _{i, j} \| x^{i}(k)-$ $P_{X_{j}}\left(x^{i}(k)\right) \| \geq \bar{\omega}$ for all $i \neq j$ and sufficiently small $\epsilon$. Letting $\omega<\bar{\omega}$, we have $\max _{i, j}\left\|x^{i}(k)-x^{j}(k)\right\|>\| x^{i}(k)-$ $x^{j}(k) \|$ for all $i, j$ and all $l_{P}+N_{P}-(n+2) M-2<k<$ $\bar{k}-(n+2) M-2$, which is a contradiction. Thus, $D_{1}=0$ and hence $\lim _{k \rightarrow+\infty}\left\|x^{i}(k)-P_{X}\left(x^{i}(k)\right)\right\|=0$ for all $i$.

Lemma 12 Under Assumptions 1, 2, 3(a), 4, 5 and 6,

$\lim _{k \rightarrow+\infty}\left\|\varphi^{i}(k)\right\|=0$ for all $i$.

Proof: Under Assumptions 1, 2, 3(a), 4, 5 and 6, $\lim _{k \rightarrow+\infty}\left\|x^{i}(k)-P_{X}\left(x^{i}(k)\right)\right\|=0$ for all $i, j$ from Lemma 11 and $\lim _{k \rightarrow+\infty} \alpha_{k}=0$. Taking the limits of both sides of (6), it follows that $\lim _{k \rightarrow+\infty}\left\|\varphi^{i}(k)\right\|=0$ for all $i$.

Proposition 1 Suppose that Assumptions 1, 2, 3(a), 4, 5 and 6 hold. Then for all $i, j, \lim _{k \rightarrow+\infty}\left\|x^{i}(k)-P_{X}\left(x_{\text {ave }}(k)\right)\right\|=$ $\lim _{k \rightarrow+\infty}\left\|v^{i}(k)-P_{X}\left(x_{\text {ave }}(k)\right)\right\|=\lim _{k \rightarrow+\infty} \| x^{i}(k)-$ $x^{j}(k) \|=0$.

Proof: Under Assumptions 1, 2, 3(a), 4, 5 and 6, Lemma 12 holds. Similar to the proof of Lemma 8, it can be proved that $\lim _{k \rightarrow+\infty}\left\|x^{i}(k)-x_{\text {ave }}(k)\right\|=$ $\lim _{k \rightarrow+\infty}\left\|x^{i}(k)-x^{j}(k)\right\|=0$ for all $i, j$ by applying Lemma 12. Since $x^{i}(k) \in X_{i}$ for all $i \in \mathcal{I}$, it follows from the definition of the projection operator that $\left\|x_{\text {ave }}(k)-P_{X_{i}}\left(x_{\text {ave }}(k)\right)\right\| \leq\left\|x_{\text {ave }}(k)-x^{i}(k)\right\|$. Thus, $\lim _{k \rightarrow+\infty}\left\|x_{\text {ave }}(k)-P_{X_{i}}\left(x_{\text {ave }}(k)\right)\right\|=0$ for all $i \in \mathcal{I}$. From Assumption 5, $x^{i}(k)$ is bounded for al$1 i \in \mathcal{I}$. Thus, under Assumption 1, it follows from Lemma 3 that $\lim _{k \rightarrow+\infty}\left\|x_{\text {ave }}(k)-P_{X}\left(x_{\text {ave }}(k)\right)\right\|=$ $\lim _{k \rightarrow+\infty}\left\|x^{i}(k)-P_{X}\left(x_{\text {ave }}(k)\right)\right\|=0$. Furthermore, since $\left\|v^{i}(k)-P_{X}\left(x_{\text {ave }}(k)\right)\right\| \leq \sum_{j=1}^{n}[\Psi(k, k)]_{j}^{i} \times$ $\left\|x^{j}(k)-P_{X}\left(x_{\text {ave }}(k)\right)\right\| \leq \sum_{j=1}^{n}\left\|x^{j}(k)-P_{X}\left(x_{\text {ave }}(k)\right)\right\|$, $\lim _{k \rightarrow+\infty}\left\|v^{i}(k)-P_{X}\left(x_{\text {ave }}(k)\right)\right\|=0$ for all $i$.

Lemma 13 Suppose that the graph $\mathcal{G}$ is fixed and strongly connected, the adjacency matrix $\mathcal{A}$ is constant, and Assumption 3(a) holds. Then we have $\lim _{k \rightarrow+\infty}\left[\mathcal{A}^{k}\right]_{j}^{i}=q_{j}>0$, where $q_{j}$ is defined in Theorem 1.

Proof: See [17].

Proof of Theorem 1: Let $\hat{z}^{*}$ be the optimal point such that $\sum_{j=1}^{n} q_{j} f_{j}\left(\hat{z}^{*}\right)=\min _{x \in X} \sum_{j=1}^{n} q_{j} f_{j}(x)$. Then for any $x \in X$, we have $\sum_{j=1}^{n} q_{j}\left[f_{j}(x)-f_{j}\left(\hat{z}^{*}\right)\right] \geq 0$. Under Assumptions 1 and 5, it follows from Lemmas 1 and 4 that, 
for all $i$ and all $k$,

$$
\begin{aligned}
& \left\|x^{i}(k+1)-\hat{z}^{*}\right\|^{2} \\
\leq & \left\|v^{i}(k)-\alpha_{k} d_{i}(k)-\hat{z}^{*}\right\|^{2}-\left\|\varphi^{i}(k)\right\|^{2} \\
= & \left\|v^{i}(k)-\hat{z}^{*}\right\|^{2}+\alpha_{k}^{2}\left\|d_{i}(k)\right\|^{2} \\
- & 2 \alpha_{k} d_{i}(k)^{T}\left(v^{i}(k)-\hat{z}^{*}\right)-\left\|\varphi^{i}(k)\right\|^{2} \\
\leq & \left\|v^{i}(k)-\hat{z}^{*}\right\|^{2}+\alpha_{k}^{2} L^{2}-2 \alpha_{k}\left(f_{i}\left(v^{i}(k)\right)-f_{i}\left(\hat{z}^{*}\right)\right) .
\end{aligned}
$$

Note that $\left\|v^{i}(k)-\hat{z}^{*}\right\|^{2} \leq \sum_{j \in N_{i}} a_{j}^{i}\left\|x^{j}(k)-\hat{z}^{*}\right\|^{2}$ for all $i$ and all $k$ from the definition of convex functions. Thus, for all $i$ and all $k$, we have $\left\|x^{i}(k+1)-\hat{z}^{*}\right\|^{2} \leq$ $\sum_{j \in N_{i}} a_{j}^{i}\left\|x^{j}(k)-\hat{z}^{*}\right\|^{2}+\alpha_{k}^{2} L^{2}-2 \alpha_{k}\left(f_{i}\left(v^{i}(k)\right)-f_{i}\left(\hat{z}^{*}\right)\right)$. Thus, the relation between $x^{i}(k+1)$ and $x^{j}(s)$ for all $k>s$ and all $i, j$ can be given by

$$
\begin{aligned}
& \left\|x^{i}(k+1)-\hat{z}^{*}\right\|^{2} \\
\leq & \sum_{j=1}^{n}\left[\mathcal{A}^{k+1-s}\right]_{j}^{i}\left\|x^{j}(s)-\hat{z}^{*}\right\|^{2}+\sum_{r=s}^{k} \alpha_{r}^{2} L^{2} \\
- & 2 \sum_{r=s+1}^{k} \sum_{j=1}^{n}\left[\mathcal{A}^{k+1-r}\right]_{j}^{i} \alpha_{r-1}\left(f_{j}\left(v^{i}(r-1)\right)\right. \\
- & \left.f_{j}\left(P_{X}\left(x_{\mathrm{ave}}(r-1)\right)\right)\right) \\
- & 2 \alpha_{k}\left(f_{i}\left(v^{i}(k)\right)-f_{i}\left(P_{X}\left(x_{\mathrm{ave}}(k)\right)\right)\right) \\
- & 2 \alpha_{k}\left(f_{i}\left(P_{X}\left(x_{\mathrm{ave}}(k)\right)\right)-f_{i}\left(\hat{z}^{*}\right)\right) \\
- & 2 \sum_{r=s+1}^{k} \sum_{j=1}^{n}\left[\mathcal{A}^{k+1-r}\right]_{j}^{i} \alpha_{r-1}\left(f_{j}\left(P_{X}\left(x_{\mathrm{ave}}(r-1)\right)\right)\right. \\
- & \left.f_{j}\left(\hat{z}^{*}\right)\right) .
\end{aligned}
$$

From Assumption 6, $\lim _{k \rightarrow+\infty} \alpha_{k}=0$. Under the assumptions of this theorem, Proposition 1 holds. Then, there exists an integer $k_{N}>0$ for any $\epsilon>0$ such that $\left\|x^{j}(k)-x^{i}(k)\right\|<$ $\epsilon,\left\|v^{i}(k)-P_{X}\left(x_{\text {ave }}(k)\right)\right\|<\frac{\epsilon}{L},\left\|x^{i}(k)-P_{X}\left(x_{\text {ave }}(k)\right)\right\|<\epsilon$ and $0<\alpha_{k}<\epsilon$ for all $k>k_{N}$. From Assumption 5, Lemma 4 holds. From the definition of the subgradient, it is easy to see that $\| f_{i}\left(v^{i}(k)\right)-f_{i}\left(P_{X}\left(x_{\text {ave }}(k)\right)\|\leq L\| v^{i}(k)-\right.$ $P_{X}\left(x_{\text {ave }}(k)\right) \|<\epsilon$ for all $k>k_{N}$ and $\| f_{i}\left(P_{X}\left(x_{\text {ave }}(k)\right)-\right.$ $f_{i}\left(\hat{z}^{*}\right)\|\leq L\| P_{X}\left(x_{\text {ave }}(k)\right)-\hat{z}^{*} \| \leq 2 L H$. Note from Lemma 13 that $\phi_{j}(r)=\lim _{k \rightarrow+\infty}\left[\mathcal{A}^{k+1-r}\right]_{j}^{i}=q_{j}$ for all $r$ and all $j$. Writing $\left[\mathcal{A}^{k+1-r}\right]_{j}^{i}$ as $\left[\mathcal{A}^{k+1-r}\right]_{j}^{i}=\left[\mathcal{A}^{k+1-r}\right]_{j}^{i}-q_{j}+q_{j}$ and applying these bounds, we have for all $i$ and all $k>s>k_{N}$,

$$
\begin{aligned}
& \left\|x^{i}(k+1)-\hat{z}^{*}\right\|^{2} \\
\leq & \sum_{j=1}^{n}\left[\mathcal{A}^{k+1-s}\right]_{j}^{i}\left\|x^{j}(s)-\hat{z}^{*}\right\|^{2}+\sum_{r=s}^{k} \alpha_{r}^{2} L^{2}+4 \epsilon L H \\
+ & 2 \sum_{r=s}^{k} \sum_{j=1}^{n} \alpha_{r}\left\|v^{j}(r-1)-P_{X}\left(x_{\mathrm{ave}}(r-1)\right)\right\| L \\
+ & 4 \sum_{r=s+1}^{k} \sum_{j=1}^{n}\left|\left[\mathcal{A}^{k+1-r}\right]_{j}^{i}-q_{j}\right| L H \epsilon+2 \epsilon^{2} \\
- & 2 \sum_{r=s+1}^{k} \sum_{j=1}^{n} q_{j} \alpha_{r-1}\left(f_{j}\left(P_{X}\left(x_{\mathrm{ave}}(r-1)\right)\right)-f_{j}\left(\hat{z}^{*}\right)\right) .
\end{aligned}
$$

Under Assumptions 2, 3(a) and 4, it follows from Lemma 6 that, there exist two constants $C_{0}$ and $0<\mu<\frac{1}{n}$ such that

$$
\begin{aligned}
& \left|\left[\mathcal{A}^{k+1-r}\right]_{j}^{i}-q_{j}\right| \leq C_{0}(1-2 \mu)^{\frac{k-r}{M(n+2)}} \text {. Thus } \\
& \quad\left\|x^{i}(k+1)-\hat{z}^{*}\right\|^{2} \\
& +2 \sum_{r=s+1}^{k} \sum_{j=1}^{n} \alpha_{r-1} q_{j}\left(f_{j}\left(P_{X}\left(x_{\text {ave }}(r-1)\right)\right)-f_{j}\left(\hat{z}^{*}\right)\right) \\
& \leq \sum_{j=1}^{n}\left[\mathcal{A}^{k+1-s}\right]_{j}^{i}\left\|x^{j}(s)-\hat{z}^{*}\right\|^{2} \\
& +\frac{4 n C_{0}}{1-(1-2 \mu) \frac{1}{M n}} L H \epsilon+4 \epsilon L H+\sum_{r=s}^{k} \alpha_{r}^{2} L^{2}+2 \epsilon^{2} \\
& +2 \sum_{r=s}^{k} \sum_{j=1}^{n} \alpha_{r}\left\|v^{i}(r)-P_{X}\left(x_{\text {ave }}(r)\right)\right\| L
\end{aligned}
$$

for all $i$ and all $k>s>k_{N}$. From Assumption 6, $\sum_{r=s}^{\infty} \alpha_{r}^{2} L^{2}<+\infty$. Under the assumptions of this theorem, from Lemma 10, $2 \sum_{r=s}^{k} \sum_{j=1}^{n} \alpha_{r} \| v^{i}(r)-$ $P_{X}\left(x_{\text {ave }}(r)\right) \| L<+\infty$. It follows that

$2 \sum_{r=s+1}^{+\infty} \sum_{j=1}^{n} \alpha_{r-1} q_{j}\left(f_{j}\left(P_{X}\left(x_{\text {ave }}(r-1)\right)\right)-f_{j}\left(\hat{z}^{*}\right)\right)<+\infty$.

Note that $\sum_{j=1}^{n} q_{j} f_{j}\left(P_{X}\left(x_{\text {ave }}(k)\right)\right) \geq \sum_{j=1}^{n} q_{j} f_{j}\left(\hat{z}^{*}\right)$ from the definition of $\hat{z}^{*}$ and $\sum_{k=s+1}^{+\infty} \alpha_{k}=+\infty$ from Assumption 6 . It follows that

$$
\liminf _{k \rightarrow+\infty} \sum_{j=1}^{n} q_{j}\left(f_{j}\left(P_{X}\left(x_{\text {ave }}(k)\right)\right)-f_{j}\left(\hat{z}^{*}\right)\right)=0 .
$$

Furthermore, since $\left\|x^{j}(s)-x^{i}(s)\right\|<\epsilon$ for all $s>k_{N}$, we have $\left\|x^{j}(s)-\hat{z}^{*}\right\|^{2}=\left\|x^{i}(s)-\hat{z}^{*}+x^{j}(s)-x^{i}(s)\right\|^{2} \leq$ $\left\|x^{i}(s)-\hat{z}^{*}\right\|^{2}+\left\|x^{j}(s)-x^{i}(s)\right\|^{2}+2\left\|x^{i}(s)-\hat{z}^{*}\right\| \| x^{j}(s)-$ $x^{i}(s)\|\leq\| x^{i}(s)-\hat{z}^{*} \|^{2}+\epsilon^{2}+4 H \epsilon$ for all $s>k_{N}$ and all $i, j$. Thus, using the stochasticity of $\mathcal{A}$, by simple calculations, $\sum_{j=1}^{n}\left[\mathcal{A}^{k+1-s}\right]_{j}^{i}\left\|x^{j}(s)-\hat{z}^{*}\right\|^{2} \leq\left\|x^{i}(s)-\hat{z}^{*}\right\|^{2}+\epsilon^{2}+4 H \epsilon$ for all $s>k_{N}$. Dropping the second term on the left side of (15), we have

$$
\begin{aligned}
& \left\|x^{i}(k+1)-\hat{z}^{*}\right\|^{2} \\
\leq & \left\|x^{j}(s)-\hat{z}^{*}\right\|^{2}+3 \epsilon^{2}+\sum_{r=s}^{+\infty} \alpha_{r}^{2} L^{2} \\
+ & \frac{4 n C_{0}}{1-(1-2 \mu) \frac{1}{M n}} L H \epsilon+4 \epsilon L H \\
+ & 2 \sum_{r=s}^{k} \sum_{j=1}^{n} \alpha_{r}\left\|v^{i}(r)-P_{X}\left(x_{\mathrm{ave}}(r)\right)\right\| L+4 H \epsilon
\end{aligned}
$$

for all $i$ and all $k>s>k_{N}$. Since $\sum_{r=s}^{\infty} \alpha_{r}^{2} L^{2}<+\infty$, $2 \sum_{r=s}^{k} \sum_{j=1}^{n} \alpha_{r}\left\|v^{i}(r)-P_{X}\left(x_{\text {ave }}(r)\right)\right\| L<+\infty$ from Lemma 10, and $\epsilon$ and $k_{N}$ can be arbirarily chosen, we have

$$
\limsup _{k \rightarrow+\infty}\left\|x^{i}(k+1)-\hat{z}^{*}\right\|^{2} \leq \liminf _{s \rightarrow+\infty}\left\|x^{i}(s)-\hat{z}^{*}\right\|^{2} .
$$

This implies that the sequence $\left\{\left\|x^{i}(k)-\hat{z}^{*}\right\|^{2}\right\}$ is convergent for all $i$. From Proposition $1, \lim _{k \rightarrow+\infty} \| x^{i}(k)-$ $P_{X}\left(x_{\text {ave }}(k)\right) \|=0$ for all $i$. Thus, the sequence $\left\{\| P_{X}\left(x_{\text {ave }}(k)\right)-\right.$ $\left.\hat{z}^{*} \|^{2}\right\}$ is convergent. In view of (16), it follows that one of the limit points of $P_{X}\left(x_{\text {ave }}(k)\right)$, denoted by $z^{*}$, must optimize the weighted sum $\sum_{j=1}^{n} q_{j} f_{j}(x)$. Take 
$\hat{z}^{*}=z^{*}$. Since $\left\{\left\|P_{X}\left(x_{\text {ave }}(k)\right)-z^{*}\right\|^{2}\right\}$ is convergen$\mathrm{t}$, it follows that $\lim _{k \rightarrow+\infty}\left\|P_{X}\left(x_{\text {ave }}(k)\right)-z^{*}\right\|=0$. Since $\lim _{k \rightarrow+\infty}\left\|x^{i}(k)-P_{X}\left(x_{\text {ave }}(k)\right)\right\|=0$ for al$1 i$, then $\lim _{k \rightarrow+\infty}\left\|x^{i}(k)-z^{*}\right\|=0$. Therefore, using the algorithm (2), the multi-agent system reaches a common point $z^{*}$ as $t \rightarrow+\infty$ and $z^{*}$ satisfies that $\sum_{j=1}^{n} q_{j} f_{j}\left(z^{*}\right)=\min _{x \in X} \sum_{j=1}^{n} q_{j} f_{j}(x)$. If Assumption 3(b) further holds, then $q_{i}=\frac{1}{n}$ for all $i$ and hence $\sum_{j=1}^{n} f_{j}\left(z^{*}\right)=\min _{x \in X} \sum_{j=1}^{n} f_{j}(x)$.

Proof of Theorem 2: Under Assumption 3, we have $\mathbf{1}^{T} \Psi(k, s)=\mathbf{1}^{T}$ and $\Psi(k, s) \mathbf{1}=\mathbf{1}$ for all $k \geq s$, which implies that $\phi_{j}(k)=\frac{1}{n}$ from Lemma 6. Following the lines of the proof of Theorem 1, Theorem 2 can be proved.

\section{Distributed optimization problem with nonidentical constraints and communication delays}

In practical applications, due to packet losses resulting in retransmission and asynchronous clocks of the agents, the states received from neighbors could be outdated, which would yield communication delays. When the communication delays exist, the balance and symmetry of the communication graphs would be destroyed, which makes the existing algorithms without delays (e.g. [1-14]) no longer valid. It is important to study the distributed optimization problem in the presence of communication delays. To solve such a problem, we use the following algorithm

$$
\begin{aligned}
x^{i}(k+1) & =P_{X_{i}}\left[\sigma x^{i}(k)-\sigma x^{i}(k-\rho)\right. \\
& \left.+\sum_{j \in N_{i}(k)} a_{j}^{i}(k) x^{j}(k-\rho)-\alpha_{k} d_{i}(k)\right]
\end{aligned}
$$

with initial condition $x(s)=x(0)$ for all $s \leq 0$, where $\eta \leq \sigma \leq a_{i}^{i}(k)$ is a constant for all $i$ and all $k, \rho$ denotes an upper bound of the delays, and all other variables are defined as in (2). Here, the graph $\mathcal{G}(k)$ is used to describe the interaction graph between agents at time $k$, and the agents are assumed to be able to access their own states without delay. Each agent stores its information over the past $\rho$ time steps. The idea of the algorithm (17) is to use each agent's own state and the information having a delay of size $\rho$ to balance the influence of the communication delays under Assumption 3.

Remark 4 In the algorithm (17), we do not impose any assumption on the symmetry of the communication delays. If the graph $\mathcal{G}(k)$ is undirected and the communication delays between any pair of neighbors are identical, i.e., $\tau_{j}^{i}(k)=$ $\tau_{i}^{j}(k)$ for all $(i, j) \in \mathcal{E}$ and all $k$, we can also adopt the following algorithm without using the stored information to solve the distributed optimization problem (1):

$$
x^{i}(k+1)=P_{X_{i}}\left[\sum_{j \in N_{i}(k)} a_{j}^{i}(k) x^{j}\left(k-\tau_{j}^{i}(k)\right)-\alpha_{k} d_{i}(k)\right],
$$

where $a_{i}^{i}(k)=\tilde{\sigma}$ for all $i$ and some positive scalar $\tilde{\sigma}$ and $\tau_{j}^{i}(k)=\tau_{i}^{j}(k)$ denotes the communication delay between agents $i$ and $j$ at time $k$ for all $i, j$.

Denote $z(k)=\left[z^{1}(k), \cdots, z^{n(\rho+1)}(k)\right]^{T}=\left[x^{1}(k)^{T}, \cdots\right.$, $\left.x^{n}(k)^{T}, x^{1}(k-1)^{T}, \cdots, x^{n}(k-1)^{T}, \cdots, x^{n}(k-\rho)^{T}\right]^{T}$. Let $v^{i}(k)=\sigma x^{i}(k)-\sigma x^{i}(k-\rho)+\sum_{j \in N_{i}(k)} a_{j}^{i}(k) z^{(j+n \rho)}(k)$ for $i \in \mathcal{I}, v^{i}(k)=z^{i-n}(k)$ for $n<i \leq(\rho+1) n$, and $\varphi^{i}(k)=z^{i}(k+1)-v^{i}(k)+\alpha_{k} d_{i}(k)$ for all $i$ where $d_{i}(k)=0$ for all $n<i \leq(\rho+1) n$. Then the equation (17) can be rewritten in compact form as $z(k+1)=v(k)-\gamma(k)+\varphi(k)=\Phi(k) z(k)-$ $\gamma(k)+\varphi(k)$, where $v(k)=\left[v^{1}(k), \cdots, v^{(\rho+1) n}(k)\right]^{T}$, $\gamma(k)=\left[\alpha_{k} d_{1}(k), \cdots, \alpha_{k} d_{n(\rho+1)}(k)\right]^{T}$, and $\varphi(k)=$ $\left[\varphi^{1}(k), \cdots, \varphi^{(\rho+1) n}(k)\right]^{T}, \Phi(k)$ is the coefficient matrix of $z(k)$. Clearly, $\Phi(k)$ is a stochastic matrix. By a similar approach to Theorem 1, it can be obtained that, under Assumptions 1, 3(a), 5 and 6, the distributed weighted optimization problem can be solved in the presence of communication delays using the algorithm (17) if the graph $\mathcal{G}(k)$ is fixed and strongly connected and the adjacency matrix $\mathcal{A}$ is constant. In particular, if Assumption 3(b) is further satisfied, it is easy to see that $\Phi(k)$ has an eigenvalue at 1 with an associated left eigenvector $\left[\mathbf{1}^{\mathbf{T}},(1-\sigma) \mathbf{1}^{\mathbf{T}}, \cdots,(1-\sigma) \mathbf{1}^{\mathbf{T}}\right]^{T}$ and hence the distributed optimization problem (1) can be solved using the algorithm (17) when the graph is strongly connected and balanced.

Let $\Psi(k, s)=\Pi_{j=s}^{k} \Phi(j)=\Phi(k) \cdots \Phi(s)$ for all $k \geq s$. The system (17) can be written as

$$
\begin{aligned}
& z^{i}(k+1)=\sum_{j=1}^{(\rho+1) n}[\Psi(k, s)]_{j}^{i} z^{j}(s) \\
& -\sum_{r=s+1}^{k}\left(\sum_{j=1}^{(\rho+1) n}[\Psi(k, r)]_{j}^{i} \alpha_{r-1} d_{j}(r-1)\right)-\alpha_{k} d_{i}(k) \\
& +\sum_{r=s+1}^{k}\left(\sum_{j=1}^{(\rho+1) n}[\Psi(k, r)]_{j}^{i} \varphi^{j}(r-1)\right)+\varphi^{i}(k) .
\end{aligned}
$$

Due to the existence of communication delays, it can be checked that the diagonal entries of the transition matrix $\Psi(k, k)$ might not be positive. As a result, the analysis approach of Proposition 1 and Theorem 1 cannot be directly applied here. To use the preceding lemmas for the proof of the main results in this section, we give the following lemma. As discussed in Lemmas 5 and 6, it is shown that each column of the transition matrix $\Psi(k, s)$ converges to a certain vector with all equal positive elements exponentially, the limits of the first $n$ columns of the transition matrix $\Psi(k, s)$ are equal, and each entry of the transition matrix $\Psi(k, s)$ is positive and nonvanishing over a certain time interval.

Lemma 14 (1) Under Assumptions 2, 3(a) and 4, each $\Psi(k, s)$ is a stochastic matrix for all $k \geq s$. Each colum$\mathrm{n}$ of $\Psi(k, s),[\Psi(k, s)]_{j}$, converges to a vector $\phi_{j}(s) \mathbf{1}$ as $k \rightarrow+\infty$, i.e., $\lim _{k \rightarrow+\infty}\left([\Psi(k, s)]_{j}-\phi_{j}(s) \mathbf{1}\right)=0$ for all $j$ where $\phi_{j}(s)>0$ and $\sum_{j=1}^{n(\rho+1)} \phi_{j}(s)=1$.

(2) Under Assumptions 2-4, $\phi_{j}(s)=\phi_{i}(s)$ for all $s$ and all $i, j \in \mathcal{I}$. 
(3) Under Assumptions 2, 3(a) and 4, there exists a constant $0<\mu<\frac{1}{n}$ such that $\left[\Psi\left(p_{2}, p_{1}\right)\right]_{j}^{i}>\mu$ for all $i$, all $j \in \mathcal{I}$ and any $p_{2}-p_{1} \geq(\rho+1) n M+2 M$.

Proof: (1) The proof can be found in [18-20] and is omitted.

(2) Break the transition matrix $\Psi(k, s)$ evenly into $(\rho+1)^{2}$ $n \times n$ square blocks for all $k \geq s$. It can be checked that under Assumption 3 the row and column sums of each $n \times n$ square block of $\Psi(k, k)$ are equal. Note that the product of any two such blocks is still a matrix having equal row and column sums. It follows that each $n \times n$ square block of $\Psi(k, k-1)$ and hence $\Psi(k, s)$ for all $k \geq s$ has equal row and column sums. In particular, consider the first block of $\Psi(k, s)$ and let $k \rightarrow+\infty$. It is easy to see that $\sum_{j=1}^{n} \phi_{j}(s)=n \phi_{i}(s)$ for all $i \in \mathcal{I}$, i.e., $\phi_{j}(s)=\phi_{i}(s)$ for all $i, j \in \mathcal{I}$.

(3) Since $k_{j+1}-k_{j} \leq M$ for all $j$ under Assumption 4 and $p_{2}-p_{1} \geq(\rho+1) n M+2 M$, there must exist integers $m \geq 0, l_{1} \geq 0$ and $l_{2} \geq 0$ such that $p_{1}=k_{m}-l_{1}$ and $p_{2}=k_{m+(\rho+1) n}+l_{2}$. Let $\overline{\mathcal{G}}(k, s)$ denote the graph whose adjacency matrix is the transition matrix $\Psi(k, s)$. It is clear that there is an edge from the $(i-1) n+j$ th node to the $i n+j$ th node in $\overline{\mathcal{G}}(k, k)$ for all $i \in\{1, \cdots, \rho\}, j \in \mathcal{I}$ and all $k$. Consider the graphs $\overline{\mathcal{G}}\left(k_{m+1}-1, p_{1}\right), \overline{\mathcal{G}}\left(k_{m+2}-1, k_{m+1}\right), \cdots$, $\overline{\mathcal{G}}\left(k_{(\rho+1) n+m-1}-1, k_{(\rho+1) n+m-2}\right), \overline{\mathcal{G}}\left(p_{2}, k_{(\rho+1) n+m-1}\right)$. Since $a_{i}^{i}(k) \geq \eta$ for all $i \in \mathcal{I}$ and all $k$ from Assumption 2, under Assumption 4, in each graph, there is a directed path of length no larger than $n(\rho+1)-1$ from the $j$ th node to the $i$ th node in each graph $\overline{\mathcal{G}}\left(k_{m+1}-1, k_{m}\right)$ for all $i$ and all $j \in \mathcal{I}$. Thus, from graph theory, there is an edge from the $j$ th node to the $i$ th node in the graph $\overline{\mathcal{G}}\left(p_{2}, p_{1}\right)$ for all $i$ and all $j \in \mathcal{I}$. That is, $\left[\Psi\left(p_{2}, p_{1}\right)\right]_{j}^{i}>0$ for all $i$ and all $j \in \mathcal{I}$. From Assumptions 2 and 3(a), each nonzero $[\Psi(k, k)]_{j}^{i}$ satisfies that $\eta \leq[\Psi(k, k)]_{j}^{i} \leq 1$ for all $k$. As the matrix $\Psi\left(p_{2}, p_{1}\right)$ is the product of a finite number of matrices $\Psi(k, k)$ from $k=p_{1}$ to $k=p_{2}$, there exists a constant $0<\mu<1$ such that $\left[\Psi\left(p_{2}, p_{1}\right)\right]_{j}^{i}>\mu$ for all $i$ and al$1 j \in \mathcal{I}$. Moreover, from the stochasticity of $\Psi\left(p_{2}, p_{1}\right)$, we have $\left.n \mu<\sum_{j=1}^{n} \Psi\left(p_{2}, p_{1}\right)\right]_{j}^{i}<1$. Hence, $\mu$ should further satisfy that $0<\mu<\frac{1}{n}$.

Proposition 2 Suppose that Assumptions 1, 2, 3(a), 4, 5 and 6 hold. For all $i, j, \lim _{k \rightarrow+\infty}\left\|x^{i}(k)-P_{X}\left(x_{\text {ave }}(k)\right)\right\|=$ $\lim _{k \rightarrow+\infty}\left\|v^{i}(k)-x_{\text {ave }}(k)\right\|=\lim _{k \rightarrow+\infty} \| x^{i}(k)-$ $x^{j}(k) \|=0$, where $x_{\text {ave }}(k)$ is defined in Lemma 10 .

Proof: Under the assumptions of this proposition, based on Lemma 14, Proposition 2 can be proved through analyzing the transition matrix $\Psi(k, s)$ and its corresponding graph $\overline{\mathcal{G}}(k, s)$ following the lines of the proof of Proposition 1 . However, it should be emphasized that when estimating the lower bound of the distances from the nodes in $\overline{\mathcal{G}}(k, s)$ to the intersection set $X$ as in the proof of Lemma 11, we only need consider the first $n$ nodes instead of all.

Remark 5 Propositions 1 and 2 show that all agents reach an agreement in the intersection of all constraint sets. The communication graphs are directed, dynamically changing, and not necessarily balanced, where only the union of the graphs is assumed to be strongly connected. It should be pointed out that the coexistence of the nonlinear projection operator, the local subgradient term, unbalanced graphs, and communication delays poses unique theoretical challenges in the analysis. In particular, symmetry would be lost in the presence of unbalanced graphs and communication delays, rendering the approaches in $[2,5,6]$ not applicable.

Our main results for the case when there exist communication delays are given in the following theorem.

Theorem 3 Suppose that Assumptions 1-6 hold. Using the algorithm (17), the multi-agent system reaches a common point $z^{*}$ in $X$ as $t \rightarrow+\infty$ and $z^{*}$ satisfies that $\sum_{j=1}^{n} f_{j}\left(z^{*}\right)=\min _{x \in X} \sum_{j=1}^{n} f_{j}(x)$.

Proof: Let $\hat{z}^{*}$ be the optimal point such that $\sum_{j=1}^{n} f_{j}\left(\hat{z}^{*}\right)=$ $\min _{x \in X} \sum_{j=1}^{n} f_{j}(x)$. From the definition of $z^{i}(k)$, for all $n<i \leq(\rho+1) n$ and all $k,\left\|z^{i}(k+1)-\hat{z}^{*}\right\|^{2}=\| z^{i-n}(k)-$ $\hat{z}^{*} \|^{2}$. Similar to the proof of Theorem 1 , for all $i \in \mathcal{I}$ and all $k$, the relation between $z^{i}(k+1)$ and $z^{j}(s)$ for all $k>s$ and all $i \in \mathcal{I}$ and $j$, can be given by

$$
\begin{aligned}
& \left\|z^{i}(k+1)-\hat{z}^{*}\right\|^{2} \\
\leq & \sum_{j=1}^{n(\rho+1)}[\Psi(k, s)]_{j}^{i}\left\|z^{j}(s)-\hat{z}^{*}\right\|^{2}+\sum_{r=s}^{k} \alpha_{r}^{2} L^{2} \\
- & 2 \sum_{r=s+1}^{k} \sum_{j=1}^{n}[\Psi(k, r)]_{j}^{i} \alpha_{r-1}\left(f_{j}\left(v^{i}(r-1)\right)\right. \\
- & \left.f_{j}\left(P_{X}\left(x_{\text {ave }}(r-1)\right)\right)\right) \\
- & 2 \alpha_{k}\left(f_{i}\left(v^{i}(k)\right)-f_{i}\left(P_{X}\left(x_{\text {ave }}(k)\right)\right)\right) \\
- & 2 \alpha_{k}\left(f_{i}\left(P_{X}\left(x_{\text {ave }}(k)\right)\right)-f_{i}\left(\hat{z}^{*}\right)\right) \\
- & 2 \sum_{r=s+1}^{k} \sum_{j=1}^{n}[\Psi(k, r)]_{j}^{i} \\
\times & \alpha_{r-1}\left(f_{j}\left(P_{X}\left(x_{\text {ave }}(r-1)\right)\right)-f_{j}\left(\hat{z}^{*}\right)\right) .
\end{aligned}
$$

Under the assumptions of this theorem, from Lemma 14, we have $0<\phi_{i}(r)=\phi_{j}(r)<1$ for all $i, j \in \mathcal{I}$ and there exists a constant $\mu>0$ such that $[\Psi(k, r)]_{j}^{i}>\mu$ for $k-r \geq(\rho+1) n M+2 M$, all $i$ and all $j \in \mathcal{I}$. By a similar analysis approach to the proof of Lemma 6(b), we have $\phi_{j}(r) \geq \min _{i}\left\{[\Psi(k, r)]_{j}^{i}\right\}>\mu$ for all $r$ and all $j \in \mathcal{I}$. Following similar steps to the proof of Theorem 1, this theorem can be proved.

\section{Numerical Examples}

In this section, we give numerical examples to illustrate the obtained results. Let $X_{1}=\{y \mid\|y\| \leq 2\}$, $X_{2}=\left\{y=\left[y_{1}, y_{2}\right]^{T}|| y_{1}-1|\leq 2 \&| y_{2}+1 \mid \leq 2\right\}$, $X_{3}=\left\{y=\left[y_{1}, y_{2}\right]^{T}|| y_{1}+1|\leq \overline{1} \&| y_{2}-1 \mid \leq 1\right\}$ and $X_{4}=\left\{y \mid\left\|y-[-3,0]^{T}\right\| \leq 3\right\}$ be four closed convex sets in the plane. Consider a multi-agent system consist- 


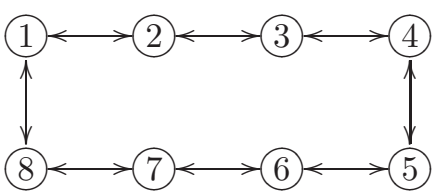

Fig. 1. The communication graph.

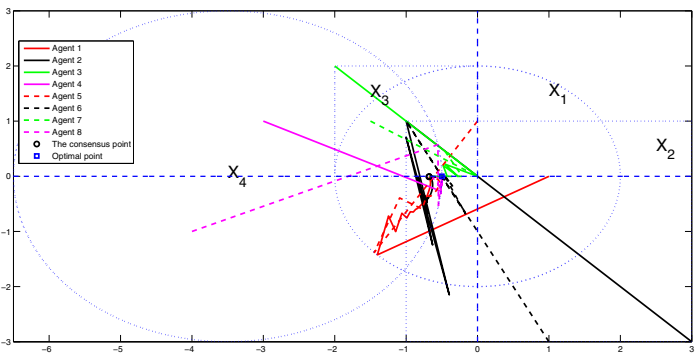

Fig. 2. State trajectories of all agents in Case I.

ing of 8 agents ${ }^{3}$. The states of agents are constrained to lie in the four closed convex sets, $X_{1}, X_{2}, X_{3}$ and $X_{4}$. Specifically, $x^{1}, x^{5} \in X_{1}, x^{2}, x^{6} \in X_{2}, x^{3}, x^{7} \in X_{3}$, and $x^{4}, x^{8} \in X_{4}$. The local objective functions are $f_{1}\left(x^{1}\right)=3 e^{1_{2}^{T} x^{1}}, f_{2}\left(x^{2}\right)=\left\|x^{2}\right\|^{2}, f_{3}\left(x^{3}\right)=\left\|x^{3}\right\|^{2}$, $f_{4}\left(x^{4}\right)=\left\|x^{4}\right\|^{2}, f_{5}\left(x^{5}\right)=7 e^{\mathbf{1}_{2}^{T} x^{5}}, f_{6}\left(x^{6}\right)=\left\|x^{6}\right\|^{2}$, $f_{7}\left(x^{7}\right)=\left\|x^{7}\right\|^{2}$ and $f_{8}\left(x^{8}\right)=\left\|x^{8}\right\|^{2}$. By simple calculations, when $x^{*} \approx[0,-0.5]^{T}$, the objective function $\sum_{i=1}^{8} f_{i}(x)$ gets its minimum value, denoted by $f^{*}$, in $X$. In the following, we give simulation results in three cases: fixed general strongly connected graphs (Case I), dynamically changing graphs with doubly stochastic adjacency matrices (Case II) and dynamically changing graphs with communication delays (Case III), where the step sizes of the algorithms (2) and (17) are taken as $\alpha_{k}=\frac{1}{1+2 k}$ for all $k$.

We first show the simulation result in Case I. The communication graph is shown in Fig. 1. The weight of each edge connecting different agents is 0.3 except $a_{2}^{1}=0.1$ and $a_{1}^{2}=0.6$ and $a_{i}^{i}$ is selected such that the adjacency matrix is stochastic. Fig. 2 shows the state trajectories of all agents. Clearly, all agents remain in their corresponding convex sets and reach a consensus in $X=\bigcap_{i=1}^{4} X_{i}$, but the consensus value is not equal to $x^{*}$. That is, the sum of the local objective functions is not minimized. However, after some calculations, it is obtained that a weighted sum of the local objective functions is minimized instead. This is consistent with Theorem 1.

Now, we show the simulation results of Cases II and III. Each agent exchanges information with at most two agents

3 Due to the distributed nature of the algorithms (only local neighbors' information is used), the algorithms work well for a large number of agents. To avoid messy trajectories when a large number of agents are involved, we have chosen only 8 agents to illustrate the theoretical results.

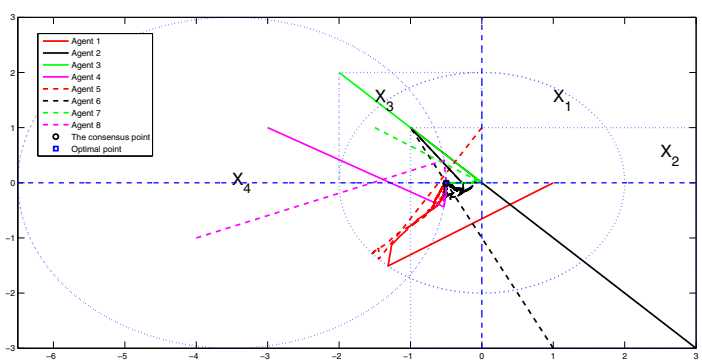

Fig. 3. State trajectories of all agents in Case II.

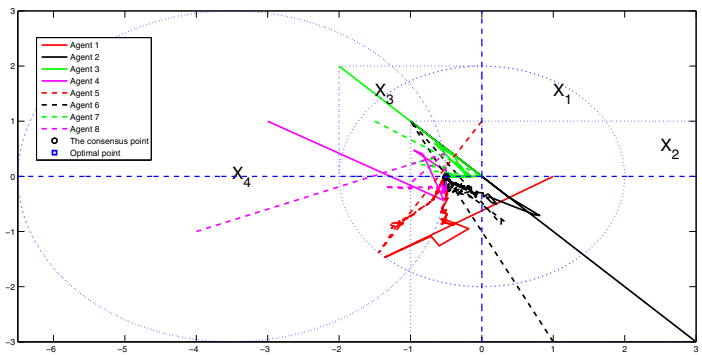

Fig. 4. State trajectories of all agents in Case III.

at each time and the edge weights between different neighbours are equal, denoted by $w$. Take $w=0.1$. The communication topologies are randomly switched as defined in Assumption 4. The union of all possible communication graphs is a closed ring as shown in Fig. 1. The state trajectories of all agents in Case II are shown in Fig. 3. Clearly, all agents converge to the point $x^{*} \approx[0,-0.5]^{T}$ while remaining in their corresponding convex sets. This is consistent with Theorem 2. In Case III, the largest delay is 4 and the parameter $\sigma$ is taken as $\sigma=0.6$. Fig. 4 shows the state trajectories of all agents. All agents also reach a consensus at $x^{*} \approx[0,-0.5]^{T}$ while remaining in their corresponding convex sets, which is consistent with Theorem 3.

Table I: The convergence time for different parameters

\begin{tabular}{|l|l|l|l|l|l|}
\hline & $\begin{array}{c}\sigma=0.6, \\
w=0.1\end{array}$ & $\begin{array}{c}\sigma=0.4, \\
w=0.1\end{array}$ & $\begin{array}{c}\sigma=0.4, \\
w=0.3\end{array}$ & $\begin{array}{c}\sigma=0.2, \\
w=0.3\end{array}$ & $\begin{array}{c}\sigma=0.9, \\
w=0.3\end{array}$ \\
\hline$\rho=0$ & 280 & 280 & 90 & 100 & 90 \\
\hline$\rho=5$ & 300 & 340 & 110 & 140 & unstable \\
\hline$\rho=10$ & 350 & 470 & 160 & 380 & unstable \\
\hline
\end{tabular}

In addition, we have done simulations to study the effects of the parameters $\sigma, \rho, w$ on the convergence time of the system in Case III. Table I shows the convergence time to the region where $\left|\sum_{i=1}^{8} f_{i}\left(x^{i}(k)\right)-f^{*}\right|<0.1$ and $\left|x^{i}(k)-x^{*}\right|<$ 0.035 for all $i$ when the parameters $\sigma, \rho, w$ take different values. It can be observed that the convergence time is not sensitive to the parameter $\sigma$ as long as $\sigma$ is lower bounded by a positive constant and no larger than $a_{i}^{i}(k)$ for all $i$. It can also be observed that the larger $w$ is and the smaller $\rho$ is, the faster the convergence rate is. However, when $w$ is too large to make $a_{i}^{i}(k)$ smaller than $\sigma$, the system may become unstable. There is a trade off between the choices 
of the parameters for the system performance.

\section{Conclusions}

In this paper, a distributed subgradient projection algorithm of multi-agent optimization with switching topologies and communication delays is considered, where each agent is constrained to stay in a closed convex set. For fixed general directed graphs, we show that consensus can be reached in the intersection set of all closed convex sets but the consensus value may not minimize the desired optimal function. Instead, the agents optimize a weighted average of the local objective functions. For the graphs whose edge weight matrices are doubly stochastic, we prove that distributed optimization can be solved when the union of the graphs is strongly connected among each time interval of certain bounded length. For switching graphs with communication delays, we show that distributed optimization can be solved by introducing additional delays to the subgradient projection algorithm and the communication delays can be arbitrarily bounded.

\section{References}

[1] A. Nedić, A. Ozdaglar. "Distributed Subgradient Methods for Multiagent Optimization", IEEE Transactions on Automatic Control, vol. 54, no. 1, 48-61, 2009.

[2] A. Nedić, A. Ozdaglar, P. A. Parrilo, "Constrained consensus and optimization in multi-agent networks", IEEE Transactions on Automatic Control, vol. 55, no. 4, pp.922-938, 2010.

[3] I. Matei and J.S. Baras, "Performance evaluation of the consensusbased distributed subgradient method under random communication topologies", IEEE Journal of Selected Topics in Signal Processing, vol. 5, no. 4, pp. 754-771, 2011.

[4] S. S. Ram, A. Nedić and V. V. Veeravalli, "Distributed Stochastic Subgradient Projection Algorithms for Convex Optimization," Journal of Optimization Theory and Applications, vol. 147, no. 3, pp. 516545,2010

[5] I. Lobel, A. Ozdaglar, and D. Feijer "Distributed multi-agent optimization with state-dependent communication", Mathematical Programming, vol. 129, no. 2, pp. 255-284, 2011.

[6] K. Srivastava, A. Nedić, "Distributed Asynchronous Constrained Stochastic Optimization", IEEE Journal of Selected Topics in Signal Processing, vol.5, no.4, pp.772-790, 2011.

[7] J. C. Duchi, A. Agarwal and M. J. Wainwright, "Dual Averaging for Distributed Optimization: Convergence Analysis and Network Scaling," IEEE Transactions on Automatic Control, vol. 57, no. 3, pp. 592-606, 2012.

[8] E. Wei, A. Ozdaglar and A. Jadbabaie, " A Distributed Newton Method for Network Utility Maximization," in Proceedings of the 50th IEEE Conference on Decision and Control and European Control Conference, Orlando, FL, December 2011, pp. 6612-6617.

[9] A. Nedić, "Asynchronous Broadcast-Based Convex Optimization Over a Network", IEEE Transactions on Automatic Control, vol. 56, no. 6, pp. 1337-1351, 2011.

[10] M. Zhu and Sonia Martínez, "An approximate dual subgradient algorithm for distributed non-convex constrained optimization", in Proceedings of IEEE Conference on Decision and Control, Atlanta, GEO, December 2010, pp. 7487-7492.
[11] D. Yuan, S. Xu, and H. Zhao, "Distributed Primal-Dual Subgradient Method for Multiagent Optimization via Consensus Algorithms", IEEE Transactions on Systems, Man and Cybernetics Part-B: Cybernetics, vol. 41, no. 6, pp. 1715-1724, 2011.

[12] B. Johansson, T. Keviczky, M. Johansson, K. H. Johansson, "A simple peer-to-peer algorithm for distributed optimization in sensor networks," in Proceedings of IEEE Conference on Decision and Control, New Orleans, LA, December 2007, pp. 4705-4710.

[13] S. S. Ram, A. Nedić and V. Veeravalli, "Incremental stochastic subgradient algorithms for convex optimization," SIAM Journal of Optimization, vol. 20, no. 2, pp. 691-717, 2009.

[14] J. Lu, C. Y. Tang, P. Regier and T. D. Bow, " Gossip Algorithms for Convex Consensus Optimization Over Networks," IEEE Transactions on Automatic Control, vol. 56, no. 12, pp. 2917-2923. 2011.

[15] P. Lin and W. Ren, "Distributed Subgradient Projection Algorithm for Multi-agent Optimization With Nonidentical Constraints and Switching Topologies," in Proceedings of IEEE Conference on Decision and Control, Maui, HI, December 2012, pp. 6813-6818

[16] C. Godsil and G. Royle, Algebraic Graph Theory, New York: Springer-Verlag, 2001.

[17] R. A. Horn and C. R. Johnson, Matrix Analysis, Cambridge, U.K. Cambridge University Press, 1987.

[18] A. Nedić and A. Ozdaglar,"Convergence Rate for Consensus with Delays", Journal of Global Optimization, vol. 47, no. 3, pp. 437-456, 2010.

[19] M. Cao, A. S. Morse, and B. D. O. Anderson,"Reaching an agreement using delayed information", in Proceedings of IEEE Conference on Decision and Control, San Diego, CA, December 2006, pp. 33753380.

[20] F. Xiao and L. Wang, "State consensus for multi-agent systems with switching topologies and time-varying delays", International Journal of Control, vol. 79, no. 10, pp. 1277-1284, 2006.

[21] D. P. Bertsekas, A. Nedić and A. E. Ozdaglar, Convex Analysis and Optimization, Belmont, MA: Athena Scientific, 2003. 